\title{
SIMULATIONS OF ACCRETION POWERED SUPERNOVAE IN THE PROGENITORS OF GAMMA-RAY BURSTS
}

\author{
Christopher C. Lindner ${ }^{1}$, Miloš Milosavljević, Rongfeng Shen ${ }^{2}$, And Pawan Kumar \\ Department of Astronomy, University of Texas, 1 University Station C1400, Austin, TX 78712, USA \\ Received 2011 August 5; accepted 2012 March 19; published 2012 April 26
}

\begin{abstract}
Observational evidence suggests a link between long-duration gamma-ray bursts (LGRBs) and Type Ic supernovae. Here, we propose a potential mechanism for Type Ic supernovae in LGRB progenitors powered solely by accretion energy. We present spherically symmetric hydrodynamic simulations of the long-term accretion of a rotating gamma-ray burst progenitor star, a "collapsar," onto the central compact object, which we take to be a black hole. The simulations were carried out with the adaptive mesh refinement code FLASH in one spatial dimension and with rotation, an explicit shear viscosity, and convection in the mixing length theory approximation. Once the accretion flow becomes rotationally supported outside of the black hole, an accretion shock forms and traverses the stellar envelope. Energy is carried from the central geometrically thick accretion disk to the stellar envelope by convection. Energy losses through neutrino emission and nuclear photodisintegration are calculated but do not seem important following the rapid early drop of the accretion rate following circularization. We find that the shock velocity, energy, and unbound mass are sensitive to convective efficiency, effective viscosity, and initial stellar angular momentum. Our simulations show that given the appropriate combinations of stellar and physical parameters, explosions with energies $\sim 5 \times 10^{50} \mathrm{erg}$, velocities $\sim 3000 \mathrm{~km} \mathrm{~s}^{-1}$, and unbound material masses $\gtrsim 6 M_{\odot}$ are possible in a rapidly rotating $16 M_{\odot}$ main-sequence progenitor star. Further work is needed to constrain the values of these parameters, to identify the likely outcomes in more plausible and massive LRGB progenitors, and to explore nucleosynthetic implications.
\end{abstract}

Key words: accretion, accretion disks - black hole physics - gamma-ray burst: general - stars: winds, outflows supernovae: general

Online-only material: color figures

\section{INTRODUCTION}

A clear observational link has been established between longduration gamma-ray bursts (LGRBs) and Type Ic supernovae (Galama et al. 1998, 2000; Reichart 1999; Bloom et al. 2002; Della Valle et al. 2003, 2006; Garnavich et al. 2003; Hjorth et al. 2003; Kawabata et al. 2003; Stanek et al. 2003; Matheson et al. 2003; Malesani et al. 2004; Campana et al. 2006; Mirabal et al. 2006; Modjaz et al. 2006; Pian et al. 2006; Chornock et al. 2010; Cobb et al. 2010; Starling et al. 2011). However, only a small percentage of Type Ic supernovae exhibit the late-time radio signatures of LGRBs (Podsiadlowski et al. 2004; Soderberg et al. 2006). LGRBs are believed to be manifestations of rotationally powered ultrarelativistic outflows developing in the wake of the formation of black holes or neutron stars in rotating progenitor. However, the exact mechanism for the production of LGRBs and their associated supernovae remains a subject of debate (Woosley \& Bloom 2006; Hjorth \& Bloom 2011, and references therein). At present, it is not clear whether the processes that give rise to LGRBs also drive stellar explosions, or whether the explosions are driven independently, perhaps by the standard, neutrino-mediated mechanism.

In the standard supernova mechanism, an outward-moving shock forms after core-bounce. This shock stalls, but may be reinvigorated by heating by neutrinos emitted during the neutronization near the proto-neutron star (Bethe \& Wilson 1985), and in principle drive the star to explosion in the so-called

\footnotetext{
1 NSF Graduate Research Fellow.

2 Department of Astronomy, \& Astrophysics, University of Toronto, 50 St. George St., Toronto, Ontario M5S 3H4, Canada.
}

delayed neutrino mechanism. Some simulations of this process in at least two spatial dimensions seem to produce successful explosions (see, e.g., Buras et al. 2006b; Scheck et al. 2006; Mezzacappa et al. 2007; Murphy \& Burrows 2008; Marek \& Janka 2009; Nordhaus et al. 2010), although the success of twodimensional and possibly three-dimensional simulations may be dependent upon the progenitor mass and the treatment of neutrinos (Buras et al. 2006a; Nordhaus et al. 2010). Supernovae associated with LGRBs seem to be more energetic than the typical Type Ic supernovae (Iwamoto et al. 1998; Woosley \& MacFadyen 1999; Mazzali et al. 2003, 2006), with large kinetic energies reaching $\sim 10^{52} \mathrm{erg}$. Even if the neutrino mechanism can unbind the star, it still seems unclear whether it can deliver the energies found in supernovae associated with LGRBs. An alternative or augmentative explosion mechanism may be required to explain the supernovae associated with LGRBs. Alternatives to the neutrino mechanism call on the extraction of the rotational energy of the central compact object-a neutron star or a black hole-or on tapping the gravitational energy of the material accreting toward the compact object. It remains to be determined which, if any, of the alternative pathways can deliver the large energies, and what are the resulting compact remnant masses.

If the post-bounce neutrino-mediated energy transfer is too weak to unbind all of the infalling stellar strata, some material may continue to fall onto the proto-neutron star and possibly take it to collapse further into a black hole (e.g., Burrows 1986; MacFadyen et al. 2001; Heger et al. 2003; Zhang et al. 2008; Sekiguchi \& Shibata 2011; O'Connor \& Ott 2010). This is especially relevant for rapidly rotating progenitors, as the progenitors with rapidly rotating cores may produce 
lower neutrino luminosities, decreasing the effectiveness of the neutrino-powered explosion mechanism (Fujimoto et al. 2006; Lee \& Ramirez-Ruiz 2006).

The infall or fallback of the stellar envelope should continue past the initial emergence of the event horizon, but then the structure of the accretion flow becomes sensitive to its angular momentum content. Given sufficient angular momentum, the flow becomes rotationally supported. Such a "collapsar" configuration has been proposed to naturally lead to the ultrarelativistic outflow in LRGBs (Woosley 1993), as gamma rays can be produced in an ultrarelativistic jet launching from the magnetosphere of the black hole that forms in the aftermath of the collapse of the rotating progenitor. The jet is powered by a continuous infall and disk-like accretion of the progenitor star's interior.

It has long been hypothesized that a "wind" outflowing from a collapsar accretion disk could unbind the stellar envelope and synthesize sufficient ${ }^{56} \mathrm{Ni}$ to produce an optically bright supernova (e.g., MacFadyen \& Woosley 1999; Pruet et al. 2003, 2004; Kohri et al. 2005). The dynamics of the energy flow in such a system has yet to be elucidated. In the present study, we utilize one-dimensional hydrodynamic simulations with rotation (1.5D) to test the hypothesis that accretion power can drive an explosion of the star. We do not simulate the core bounce, and simply posit that any prompt and neutrino-reinvigorated shock has failed and that the stellar atmosphere has not acquired outward motion and is free to accrete toward the black hole.

In Lindner et al. (2010), we simulated the post-corecollapse hydrodynamical evolution of the rapidly rotating $14 M_{\odot}$ Wolf-Rayet (W-R) stellar model 16TI of Woosley \& Heger (2006) that has been proposed as an LGRB progenitor. The rate at which the infalling stellar envelope was being accreted onto the black hole evolved through two distinct phases during the first $\sim 200 \mathrm{~s}$ following the initial collapse of the stellar core. First, the low specific angular momentum material of the inner layers of the star accreted quasi-spherically through the inner boundary and is presumed to have accreted onto the black hole. Then, the material that had sufficient angular momentum to become rotationally supported on the computational grid formed a thick accretion torus. Simultaneously, an accretion shock appeared at the innermost radii and traversed the star. Most of the stellar envelope traversed by the shock was in radial hydrostatic equilibrium and convective; convection transported the energy dissipated at the smallest simulated radii toward the expanding shock. The central accretion rate was nearly timeindependent prior to rotating torus and shock formation, and dropped sharply afterward. The abrupt drop of the accretion rate closely resembled the prompt $\gamma$-ray and the early X-ray LGRB light curves measured with the NASA Swift satellite (Tagliaferri et al. 2005; Nousek et al. 2006; O'Brien et al. 2006), adding weight to the hypothesis that the light curves are responding to an evolution of the central accretion rate (Kumar et al. 2008a, 2008b). Because the innermost simulated radius was $500 \mathrm{~km}$, much larger than the innermost stable circular orbit around the central black hole $(5-50 \mathrm{~km})$, the accreted-mass-to-energy conversion efficiency was low and the shock acquired relatively low velocities, $\sim 1000 \mathrm{~km} \mathrm{~s}^{-1}$, while in the interior of the star. The star did not explode, but only lost mass to the thermally driven wind that set in after the shock had traversed the star.

In collapsars, a substantially larger accretion energy is dissipated at the radii left out from the Lindner et al. (2010) simulations, closer to the black hole, but only a fraction of this energy couples to the stellar envelope. The rest may be lost to the emission of neutrinos and to the photodisintegration of hydrostatic elements into free nucleons as well as to advection into the black hole. Crude analytical considerations (Milosavljević et al. 2012) suggest that following shock formation and the rapid accretion rate drop seen in Lindner et al. (2010), neutrino losses are relatively small. Then, the amount of energy transferred onto the envelope is determined by the competition of the inward advective and the outward convective energy transport. The advection arises from the inward drift of the fluid in response to magnetohydrodynamic (MHD) stresses; the convection arises from entropy gradients arising from the dissipation of MHD turbulence. If convective transport is efficient, the amount of energy transferred from near the black hole to the shocked envelope can be sufficient to drive a fast shock with velocity $\gg 1000 \mathrm{~km} \mathrm{~s}^{-1}$ and unbind the star. The model of Milosavljević et al. (2012) suggests that the parameters determining the viability and energy of such accretion-powered supernovae are the viscous stress-to-pressure ratio $\alpha$ and the convective mixing length $\lambda_{\text {conv }}$. The model could not, of course, capture the consequences of the interplay of pressure and rotation at the critical radii where the two sources of radial support against gravity are comparable.

In this work, we show the results of a series of rotating onedimensional simulations of the immediate aftermath of the collapse of a rapidly rotating LGRB progenitor star's core. While one-dimensional, our simulations include rotation in a spherically averaged sense and implement a modified $\alpha$-viscosity prescription. One customarily refers to such simulations as "1.5 dimensional." They also take into account optically thin cooling by neutrino emission, cooling and heating by nuclear processes, and energy and compositional transport by convection in the mixing length theory approximation. This work is complementary to our rotating two-dimensional simulations (2.5D) of collapsar accretion (Lindner et al. 2010), in which we simulated only relatively large radii and did not incorporate nuclear and neutrino physics. Here, we sacrifice in spatial dimensionality to make it possible to track rudimentary nuclear compositional transformation and simulate smaller radii $(r>25 \mathrm{~km})$ over similarly extended time periods ( $\sim 40-100 \mathrm{~s})$. In the presence of cooling by neutrino emission the rotating central torus may be geometrically thin (e.g., MacFadyen \& Woosley 1999; Popham et al. 1999; Kohri et al. 2005; Chen \& Beloborodov 2007; Sekiguchi \& Shibata 2011; Taylor et al. 2011). Therefore, we include corrections to approximate the effects of such flow. The principal source of model uncertainty is the efficiency of convection, which in the mixing length approximation can be parameterized with an effective value of the mixing length. To our best knowledge, there has not been a systematic first principles study of convective efficiencies in the rapidly convecting regime. Thus the mixing length $\lambda_{\text {conv }}$ and the viscous shear stress-to-pressure ratio $\alpha$ are the parameter dependences that we explore.

A magnetic outflow driven by a proto-neutron star may carry an energy similar to that of a supernova (e.g., BisnovatyiKogan 1971; Wheeler et al. 2000; Thompson et al. 2004; Bucciantini et al. 2007; Burrows et al. 2007; Dessart et al. 2008). However, the outflow may be too axially collimated to produce a standard, quasi-spherical explosion (Bucciantini et al. 2008, 2009). Here, we assume that any explosion mechanism preceding the collapse into a black hole has failed. Clearly, our one-dimensional model cannot capture the effects of the formation of a magnetized jet, after an accretion disk has formed. Although this is an integral component to the collapsar model 
for LGRBs, we omit any treatment of the jet in the present work.

This work is organized as follows. In Section 2, we discuss our numerical algorithm. In Section 3, we present the results of our simulations. In Section 4, we identify the parameters critical to our model and discuss their implications for real accretion powered supernovae. Finally, in Section 5, we summarize our conclusions.

\section{NUMERICAL ALGORITHM}

The simulations were carried out with the piecewise-parabolic method (PPM) solver in the adaptive-mesh-refinement code FLASH (Fryxell et al. 2000), version 3.2, in one spatial dimension. Although the rotating stellar collapse is inherently three dimensional, we have chosen to approximate the key multidimensional effects, including angular momentum transport and convective energy and compositional transport, with a spherically averaged transport scheme. In Section 2.1, we describe our implementation of angular momentum transport. In Section 2.2, we describe our calculation of the self-gravity of the fluid. In Section 2.3, we describe our modeling of the transition toward nuclear statistical equilibrium (NSE) in the hot inner accretion flow. In Section 2.4, we discuss cooling by neutrino emission. In Section 2.5, we describe our treatment of convective energy transport and compositional mixing. In Section 2.6, we describe the corrections that we apply in situations where, in the presence of cooling, the accretion flow is expected to be geometrically thin. In Section 2.7, we describe our initial and boundary conditions. In Section 2.8, we show the results from tests of the code. Finally, in Section 2.9, we briefly review the various limitations of our method.

\subsection{Angular Momentum}

To include rotation and angular momentum transport in our one-dimensional model, we track the specific angular momentum $\ell \equiv r v_{\phi}$, where $v_{\phi}$ is the azimuthal velocity, which we interpret as the mass-weighted spherical average of an underlying polar-angle-dependent angular momentum $\ell(r, \theta)$. If, e.g., spherical shells rotate rigidly, $\ell(r, \theta) \propto \sin ^{2} \theta$, and the fluid density is spherically symmetric, then the one-dimensional specific angular momentum is two-thirds of the midplane value, $\ell=2 / 3 \ell_{\text {mid }}$. The azimuthal Navier-Stokes equation, combined with the equation of mass continuity, then implies the onedimensional angular momentum transport equation (see, e.g., Thompson et al. 2005)

$$
\frac{\partial(\rho \ell)}{\partial t}+\frac{1}{r^{2}} \frac{\partial\left(r^{2} v_{r} \rho \ell\right)}{\partial r}-\frac{1}{r^{2}} \frac{\partial}{\partial r}\left(r^{3} v \rho \sigma_{r \phi}\right)=0,
$$

where $v$ is a shear viscosity and

$$
\sigma_{r \phi}=r \frac{\partial}{\partial r}\left(\frac{\ell}{r^{2}}\right)
$$

is the $r-\phi$ component of the shear tensor. The energy dissipated through shear viscosity was accounted for by including the specific heating rate (see, e.g., Landau \& Lifshitz 1959)

$$
\dot{\epsilon}_{\mathrm{visc}} \equiv \frac{Q_{\mathrm{visc}}}{\rho}=v \sigma_{r \phi}^{2},
$$

where $Q_{\text {visc }}$ denotes the volumetric viscous heating rate. The dimensional reduction in Equation (1) is inaccurate in regions where the disk is geometrically thin. There the mass-weighted spherical average closely approximates the midplane value, $\ell \sim \ell_{\text {mid }}$. We ignore this effect, but we do incorporate thermodynamic corrections addressing the transition to a thin disk in Section 2.6.

Our treatment of shear viscosity is similar to our methodology in Lindner et al. (2010), and for completeness we reproduce our methodology here. Since we do not simulate the magnetic field of the fluid, we utilize a local definition of the shear viscosity to emulate the magnetic stress arising from the nonlinear development of the magnetorotational instability (MRI; Balbus \& Hawley 1998 and references therein). It should be kept in mind, however, that the effects of MRI are in some respects very different from those of the viscous stress. For example, the thick disk surrounding our collapsar black hole is convective; in unmagnetized accretion flows convection transports angular momentum inward, toward the center of rotation (Ryu \& Goodman 1992; Stone \& Balbus 1996; Igumenshchev et al. 2000), whereas in magnetized flows, convection can also transport angular momentum outward (Balbus \& Hawley 2002; Igumenshchev 2002; Igumenshchev et al. 2003; Christodoulou et al. 2003). Although we include treatment for convective energy flux and compositional mixing (see Section 2.5), we do not include angular momentum transport by convection.

Our definition of the local viscous stress emulating the MRI must be valid under rotationally supported, pressure supported, and freely falling conditions, and we proceed as in Lindner et al. (2010). Thompson et al. (2005) suggest that since the wavenumber of the fastest growing MRI mode, which is given by the dispersion relation $v_{\mathrm{A}} k \sim \Omega$ where $v_{A}$ is the Alfvén velocity and $\Omega=v_{\phi} / r$ is the angular velocity, should be about the gas pressure scale height in the saturated quasi-state state, $k \propto H^{-1}$, the Maxwell $\rho v_{\mathrm{A}}^{2}$ and viscous $v \rho \Omega$ stresses (up to factors in $|d \ln \Omega / d \ln r|$ that we neglect) can be equated if the viscosity is given by

$$
v_{\mathrm{MRI}}=\alpha H^{2} \Omega,
$$

where $\alpha$ is a dimensionless parameter. If the pressure scale height is defined locally,

$$
H=|\nabla \ln P|^{-1},
$$

the viscosity defined in Equation (4) suffers from divergences at pressure extrema. To alleviate this problem, as in Lindner et al. (2010), we define a second viscosity according to the Shakura \& Sunyaev (1973) prescription

$$
\nu_{\mathrm{SS}}=\alpha \frac{P}{\rho} \Omega^{-1} \text {. }
$$

Shakura-Sunyaev viscosity overestimates the magnetic stress in stratified hydrostatic atmospheres. We thus set the viscosity in Equations (1) and (3) to equal the harmonic mean of the above two viscosities

$$
v=\frac{2 v_{\mathrm{MRI}} v_{\mathrm{SS}}}{v_{\mathrm{MRI}}+v_{\mathrm{SS}}},
$$

where the pressure gradient in Equation (5) is calculated by the finite differencing of pressure in neighboring fluid cells. Additionally, we have applied a Gaussian kernel smoothing to the radial dependence of $H$ to help filter short-wavelength numerical instabilities. We describe this procedure in Section 2.5.

In FLASH, we treat specific angular momentum as a conserved "mass scalar" that is being advected with the fluid, which 
makes $\rho \ell$ a conserved variable; the corresponding centrifugal force is then incorporated in the calculation of the gravitational acceleration as we explain in Section 2.2 below. Then the third parabolic term in Equation (1) is computed explicitly through the inclusion of the radial $\rho \ell$-flux $-r v \rho \sigma_{r \phi}$ in the advection of $\ell$.

Numerical stability of an explicit treatment of a parabolic term places an upper limit on the time step

$$
\Delta t<\frac{\Delta r^{2}}{2 v}
$$

where $\Delta r$ is the grid resolution. For $\alpha \gg 0.01$, the viscous time step in our simulations becomes significantly shorter than the Courant time step. In our test integrations with a $\gamma$-law equation of state (EOS; Lindner et al. 2010), we find that, while not implying an outright instability, a choice of $\Delta t$ that saturates the limit in Equation (8) results in weak stationary staggered perturbations in the fluid variables. We ignore this complication and allow our time step to be set by the limit in Equation (8) of the cell with the smallest viscous diffusion time across the cell.

\subsection{Gravity}

We calculate contributions to the gravitational potential from a central point mass and a spherically symmetric extended envelope. General relativistic effects become important at the innermost radius, which in some simulations is as small as $r_{\text {min }}=25 \mathrm{~km}$. At radii $r \sim r_{\min }$, the black hole dominates the enclosed mass after about $0.5 \mathrm{~s}$. Thus, we describe the gravity of the black hole using the approximate, pseudoNewtonian gravitational force for a rotating black hole proposed by Artemova et al. (1996), which is a generalization of the Paczyński \& Wiita (1980) pseudopotential to rotating black holes. However, we continue to calculate the gravity of the fluid in the Newtonian limit. The Artemova et al. gravitational acceleration in the equatorial plane of a rotating black hole is given by

$$
\mathbf{g}_{\mathrm{BH}}(r, \theta=\pi / 2)=-\frac{G M_{\mathrm{BH}}}{r^{2-\beta}\left(r-r_{\mathrm{H}}\right)^{\beta}} \hat{\mathbf{r}},
$$

where $r_{\mathrm{H}}=\left[1+\left(1-a^{2}\right)^{1 / 2}\right] G M_{\mathrm{BH}} / c^{2}$ is the radius of the event horizon expressed in terms of the dimensionless spin parameter $a$, and $\beta=r_{\mathrm{ISCO}} / r_{\mathrm{H}}-1$ is a dimensionless exponent with $r_{\text {ISCO }}$ denoting radius of the innermost stable prograde equatorial circular orbit. We assume a dimensionless spin parameter of $a=0.9$ in these calculations. Our treatment does not incorporate general relativistic corrections to the viscous stress and momentum equations (see, e.g., Beloborodov 1999).

We adopt the form of the gravitational acceleration in Equation (9), which was derived for the equatorial plane of the black hole, to represent the mass-weighted spherical average of the gravitational acceleration, by setting $g_{\mathrm{BH}}(r)=g_{\mathrm{BH}}(r, \theta=$ $\pi / 2)$. This approximation is appropriate when the accreting mass is concentrated in the equatorial plane, especially when the innermost disk is geometrically thin, and is probably rather inaccurate for an accretion flow that is geometrically thick down to $r_{\text {ISCO }}$. Our simulations predict a geometrically thin disk at $r \lesssim 100 \mathrm{~km}$ or greater radii after material has circularized in our simulation, so this assumption seems adequate.
For each zone, the gravitational acceleration due to fluid selfgravity is calculated from

$$
\begin{aligned}
\mathbf{g}_{\text {self }}\left(r_{i}\right)= & -\frac{4 \pi}{3} \frac{G}{r^{2}}\left\{\rho_{i}\left[r_{i}^{3}-\left(r_{i}-\frac{\Delta r_{i}}{2}\right)^{3}\right]\right. \\
& \left.+\sum_{r_{k}<r_{i}} \rho_{k}\left[\left(r_{k}+\frac{\Delta r_{k}}{2}\right)^{3}-\left(r_{k}-\frac{\Delta r_{k}}{2}\right)^{3}\right]\right\} \hat{\mathbf{r}},
\end{aligned}
$$

where $\Delta r_{i}$ and $\Delta r_{k}$ are the radial widths of the grid cells. The net gravitational and inertial acceleration in our calculation is then given by

$$
\mathbf{a}_{\mathrm{tot}}=\mathbf{g}_{\mathrm{BH}}+\mathbf{g}_{\mathrm{self}}+\mathbf{a}_{\mathrm{cent}},
$$

where

$$
\mathbf{a}_{\mathrm{cent}}=\frac{\ell^{2}}{r^{3}} \hat{\mathbf{r}}
$$

is the centrifugal acceleration.

\subsection{Nuclear Processes and the Equation of State}

To calculate the internal energy of the fluid, we use the Helmholtz EOS of Timmes \& Swesty (2000) included with the FLASH distribution, which accounts for the contributions to pressure and other thermodynamic quantities from radiation, ions, electrons, positrons, and Coulomb corrections. We track the abundances of 47 nuclear isotopes treated in the nuclear statistical equilibrium (NSE) calculations of Seitenzahl et al. (2008) and pass the local nuclear composition to the EOS as input. Given density, temperature, and nuclear composition, the Helmholtz EOS provides the internal energy, density, pressure, entropy, specific heats, adiabatic indices, electron chemical potential, and various derivative thermodynamic quantities. During the course of the thermodynamic update and the cooling update which is operator split from the thermodynamic update, the temperature must be derived from the internal energy, and in the Helmholtz EOS this is achieved by numerically solving for the implicit relation

$$
\epsilon_{\mathrm{EOS}}(\rho, T, \mathbf{X})=\epsilon
$$

for the temperature, where $\epsilon$ is the specific internal energy and $\mathbf{X} \equiv\left(X_{1}, \ldots, X_{47}\right)$ is the vector of isotopic mass fractions $X_{i}$.

The fluid heats and cools in response to nuclear compositional transformation. We do not integrate a nuclear reaction network, but instead model the change of the nuclear composition as a gradual convergence to NSE in the part of the flow where the convergence timescale $\tau_{\mathrm{NSE}}$ is comparable to or shorter than the age of the system. In this model, as we explain below, the nuclear composition responds instantaneously to a change of the temperature, implying that the dependence of the composition on the temperature must be taken into account, in a manner that conserves the combined specific internal and nuclear energy $\epsilon+\epsilon_{\text {nuc }}$ when solving the EOS for temperature. Here, $\epsilon_{\text {nuc }}$ is the specific (negative) nuclear binding energy of the fluid

$$
\epsilon_{\mathrm{nuc}}=\sum_{i} \frac{X_{i} E_{\mathrm{B}, i}}{A_{i} m_{p}}
$$

while $E_{\mathrm{B}, i}$ is the negative nuclear binding energy of the isotope and $A_{i}$ is the atomic mass of the isotope. 
The timescale for convergence to NSE can be approximated via (Khokhlov 1991; see also Calder et al. 2007)

$$
\tau_{\mathrm{NSE}}=\rho^{0.2} \exp \left(\frac{179.7}{T_{9}}-40.5\right) \mathrm{s},
$$

where $T=10^{9} T_{9} \mathrm{~K}$ and $\rho$ is the density in $\mathrm{g} \mathrm{cm}^{-3}$. At relevant densities, this timescale is of the order of $1 \mathrm{~s}$ for $T_{\mathrm{NSE}} \approx 4 \times 10^{9} \mathrm{~K}$. We calculate the nuclear mass fractions using the publicly available solver of Seitenzahl et al. (2008) which solves for the NSE mass fractions $X_{\mathrm{NSE}, i}$ of 47 nuclear isotopes as a function of density $\rho$, temperature $T$, and protonto-nucleon ratio $Y_{e}=\sum_{i} Z_{i} X_{i} / A_{i}$, where $Z_{i}$ is the atomic number an isotope. At temperatures $T>3 \times 10^{9} \mathrm{~K}$ we model convergence to NSE via

$$
\left(\frac{\partial X_{i}}{\partial t}\right)_{\mathrm{nuc}}=\frac{X_{i, \mathrm{NSE}}\left(\rho, T_{\mathrm{NSE}}, Y_{e}\right)-X_{i}}{\tau_{\mathrm{NSE}}(\rho, T)},
$$

where $T_{\mathrm{NSE}}$ is the temperature that the fluid element would have given enough time to relax into NSE while keeping the total specific energy $\epsilon+\epsilon_{\mathrm{NSE}}$ and proton-to-nucleon ratio $Y_{e}$ fixed. The temperature $T_{\mathrm{NSE}}$ is implicitly defined by the condition (cf. Equation (13))

$$
\begin{aligned}
& \epsilon_{\mathrm{EOS}}\left[\rho, T_{\mathrm{NSE}}, \mathbf{X}_{\mathrm{NSE}}\left(\rho, T_{\mathrm{NSE}}, Y_{e}\right)\right]+\epsilon_{\mathrm{nuc}}\left[\mathbf{X}_{\mathrm{NSE}}\left(\rho, T_{\mathrm{NSE}}, Y_{e}\right)\right] \\
& \quad=\epsilon(\rho, T, \mathbf{X})+\epsilon_{\mathrm{nuc}}(\mathbf{X}) .
\end{aligned}
$$

This condition ensures that the sum of the internal and nuclear energy densities in NSE would equal the sum of the two energy densities in the model. We solve Equation (17) for $T_{\mathrm{NSE}}\left(\rho, Y_{e}, \epsilon, \epsilon_{\mathrm{nuc}}\right)$ iteratively and then update the abundances by discretizing Equation (16) with

$$
\begin{aligned}
& X_{i}(t+\Delta t)=X_{i, \mathrm{NSE}}\left(\rho, T_{\mathrm{NSE}}, Y_{e}\right) \\
& \quad+\left[X_{i}(t)-X_{i, \mathrm{NSE}}\left(\rho, T_{\mathrm{NSE}}, Y_{e}\right)\right] \exp \left[-\frac{\Delta t}{\tau_{\mathrm{NSE}}(\rho, T)}\right]
\end{aligned}
$$

Following the update of the nuclear mass fractions, we update the specific internal energy to account for heating or cooling due to any change in specific nuclear binding energy

$$
\epsilon(t+\Delta t)=\epsilon(t)+\epsilon_{\mathrm{nuc}}[\mathbf{X}(t)]-\epsilon_{\mathrm{nuc}}[\mathbf{X}(t+\Delta t)],
$$

and finally update the temperature from Equation (13).

This prescription does not affect the proton-to-nucleon ratio $Y_{e}$; that latter is a conserved mass scalar in our simulations. Thus, the expected partial neutronization in the mildly degenerate innermost segment of the accretion flow is not calculated and our prescription cannot be used to accurately estimate the ${ }^{56} \mathrm{Ni}$ fraction within the $\mathrm{Fe}$-group elements synthesized in the simulation.

\subsection{Cooling}

The hot innermost accretion flow cools via neutrino emission. At the densities observed in our simulation, the disk and stellar atmosphere are transparent to neutrinos. The two most significant neutrino-emission channels (e.g., Di Matteo et al. 2002, and references therein) are as follows.

1. Pair capture on free nucleons (the Urca process). $p+e^{-} \rightarrow$ $n+v$ and $n+e^{+} \rightarrow p+\bar{v}$. The cooling rate is

$$
Q_{e N}=9 \times 10^{33} \rho_{10} T_{11}^{6} X_{\text {nuc }} \operatorname{erg~cm}^{-3} \mathrm{~s}^{-1},
$$

where $\rho=10^{10} \rho_{10} \mathrm{~g} \mathrm{~cm}^{-3}, T=10^{11} T_{11} \mathrm{~K}$, and $X_{\text {nuc }}=$ $X_{p}+X_{n}$ is the mass fraction in free nucleons.

2. Pair annihilation $\left(e^{-}+e^{+} \longrightarrow v+\bar{v}\right)$. The cooling rate is

$$
Q_{e^{+} e^{-}}=1.5 \times 10^{33} T_{11}^{9} \mathrm{erg} \mathrm{cm}^{-3} \mathrm{~s}^{-1} .
$$

All three flavors, $e, \mu$, and $\tau$, of neutrinos are included.

We have included the above neutrino cooling rates in our calculations, where losses are computed via

$$
\epsilon(t+\Delta t)=\epsilon(t)-\frac{Q_{v}}{\rho} \Delta t,
$$

where $Q_{v}=Q_{e N}+Q_{e^{+} e^{-}}$is the total volumetric neutrino cooling rate. The update of the internal energy due to cooling is operator split from the update due to nuclear compositional change.

\subsection{Convection}

We introduce convective energy transport and compositional mixing within the framework of mixing length theory (e.g., Kuhfuß 1986). In the calculation of the convective transport fluxes, we ignore the radial variation of the mean molecular weight as well as rotation, and the condition for instability is simply the Schwarzschild criterion, $\partial s / \partial r<0$. Then, in unstable zones, the convective energy flux is

$$
F_{\text {conv }}=-\frac{1}{2} c_{P} \rho v_{\text {conv }} \lambda_{\text {conv }}\left(\frac{\partial T}{\partial s}\right)_{P} \frac{\partial s}{\partial r},
$$

where $c_{P}$ is the specific heat at constant pressure, $\lambda_{\text {conv }}$ is the length over which convection occurs, $s$ is specific entropy, and $v_{\text {conv }}$ is the convective velocity. The convective velocity can be approximated by

$$
v_{\text {conv }} \sim \frac{1}{2} \lambda_{\text {conv }}\left[-\frac{g}{\rho}\left(\frac{\partial \rho}{\partial T}\right)_{P} \frac{T}{c_{P}} \frac{d s}{d r}\right]^{1 / 2},
$$

where $g<0$ is the gravitational acceleration in the local rest frame of the convectively unstable fluid

$$
g=g_{\text {grav }}-\frac{d v}{d t}=\frac{1}{\rho} \frac{\partial P}{\partial r}=-\frac{P}{\rho H},
$$

and $g_{\text {grav }}=g_{\mathrm{BH}}+g_{\text {self }}$ is the net gravitational acceleration in the inertial frame, $v$ in the second step denotes the massweighted spherical average of the fluid velocity at radius $r$. To parameterize our uncertainty regarding the value of the convective mixing length, we introduce a dimensionless parameter $\xi_{\text {conv }} \sim \mathcal{O}(1)$ defined as

$$
\xi_{\mathrm{conv}} \equiv\left(\frac{\lambda_{\mathrm{conv}}}{H}\right)^{2}
$$

Then, combining Equations (23)-(26), we obtain the standard expression

$$
F_{\text {conv }}=\frac{1}{4} \xi_{\text {conv }} H^{2} c_{P}\left[-\frac{P}{H}\left(\frac{\partial \rho}{\partial T}\right)_{P}\right]^{1 / 2}\left(-\frac{T}{c_{P}} \frac{\partial s}{\partial r}\right)^{3 / 2}
$$

which is appropriate even when the fluid is not in hydrostatic equilibrium and $v_{r} \neq 0$.

In evaluating the convective energy flux at a boundary (face) of a computational cell, we use face-centered linear 
interpolation of the density, temperature, and pressure. The zone-centered values of the specific heat $c_{P}$, specific entropy $s$, and thermodynamic derivatives $(\partial P / \partial T)_{\rho}$ and $(\partial P / \partial \rho)_{T}$ are returned by the EOS routine, and the face-centered values are again computed by linear interpolation. Then, $(\partial \rho / \partial T)_{P}$ is calculated from

$$
\left(\frac{\partial \rho}{\partial T}\right)_{P}=-\left(\frac{\partial P}{\partial T}\right)_{\rho} /\left(\frac{\partial P}{\partial \rho}\right)_{T} .
$$

The convective energy flux never exceeds

$$
F_{\text {conv }} \leqslant \rho \epsilon c_{\mathrm{s}}
$$

where $c_{\mathrm{s}}=\left(\gamma_{c} P / \rho\right)^{1 / 2}$ is the adiabatic sound speed and $\gamma_{c}$ is the adiabatic index.

We anticipate that a local application of MLT, in which the expression for the convective energy flux contains a pressure derivative in the denominator, may contain an instability. The instability is an artifact of modeling the intrinsically nonlocal convective energy transport with a local nonlinear differential operator. To control-if not entirely prevent — undesirable outcomes of the instability, we filter short wavelength perturbations in the calculation of the pressure scale height $H$ that enters our estimates of the viscosity and the energy flux transported by convection by applying a Gaussian smoothing

$$
P_{\text {smooth }}(r)=\frac{\sum_{i} k_{i}(r) P_{i}}{\sum_{i} k_{i}(r)},
$$

where the summations are over all of the cells in the simulation, and the spherically averaged smoothing kernel $k_{i}$ is given by

$k_{i}(r)=\frac{1}{2 \sqrt{2 \pi}} \frac{\Delta r_{i} r_{i}}{r \sigma}\left\{\exp \left[-\frac{\left(r-r_{i}\right)^{2}}{2 \sigma^{2}}\right]-\exp \left[-\frac{\left(r+r_{i}\right)^{2}}{2 \sigma^{2}}\right]\right\}$.

Here, $\sigma$ is a radius-dependent smoothing length that we set to $(1 / 2) r$. Similarly, in the evaluation of the specific entropy derivative in Equation (27), we smooth the specific entropy $s$ via

$$
s_{\text {smooth }}(r)=\frac{\sum_{i} k_{i}(r) \rho_{i} s_{i}}{\sum_{i} k_{i}(r) \rho_{i}} .
$$

The filtering affects only the evaluation of $F_{\text {conv }}$ and helps avoid breakdown of our transport scheme, but residual artificial nonpropagating waves do develop, and saturate, on wavelengths comparable to the smoothing length.

The accretion shock formally presents a negative entropy gradient but physically does not give rise to convection. The upstream of the shockwave is marginally convectively stable as the shockwave traverses the progenitor's convective core, and becomes absolutely stable in the radiative envelope. To prevent spurious convection across the shock transition, we modify the convective flux to decline to zero linearly near the shock

$$
F_{\text {conv } \text { mod }}(r)= \begin{cases}\left(1-r / r_{\text {shock }}\right) F_{\text {conv }}(r), & r<r_{\text {shock }}, \\ 0, & r \geqslant r_{\text {shock }},\end{cases}
$$

where $r_{\text {shock }}$ is the radius of the accretion shock front which we track during the simulation.

Murphy \& Meakin (2011) argue that on physical grounds, in quasi-stationary "stalled" shocks in the standard core-collapse context, the distance from the shock $r_{\text {shock }}-r$ is the appropriate convective length scale near the shock, as convective eddies can grow to the largest size available to them. If we had set the convective mixing length $\lambda_{\text {conv }}$ proportional to the distance from the shock, which is the adaptation of MLT that Murphy \& Meakin suggest, Equation (33) would have contained a quadratic factor $\left(1-r / r_{\text {shock }}\right)^{2}$, instead of the linear factor $\left(1-r / r_{\text {shock }}\right)$ that we employ. The physically motivated modification of $\lambda_{\text {conv }}$ of Murphy \& Meakin, which we became aware of after the completion of this work, and our ad hoc version should give rise to similar dynamics, especially when the shock travels outward as in our simulations.

Convection also gives rise to compositional mixing in the convective region. We model the mixing of nuclear species in the diffusion approximation (e.g., Cloutman \& Eoll 1976; Kuhfuß 1986)

$$
\left[\frac{\partial\left(\rho X_{i}\right)}{\partial t}\right]_{\text {mix }}=-\frac{1}{r^{2}} \frac{\partial}{\partial r}\left(r^{2} \mathcal{F}_{\text {mix }, i}\right)
$$

where

$$
\mathcal{F}_{\text {mix }, i}=-\frac{1}{3} v_{\text {conv }} \rho \frac{\partial X_{i}}{\partial r}
$$

is the mass flux of species $i$ transported by convection, while $v_{\text {conv }}$ is the compositional diffusivity which we take to be proportional to the convective velocity multiplied by the pressure scale height

$$
v_{\mathrm{conv}}=\xi_{\mathrm{mix}} v_{\mathrm{conv}} \lambda_{\mathrm{conv}},
$$

and $\xi_{\text {mix }} \sim \mathcal{O}(1)$ is a dimensionless parameter. We again apply the flux limitation behind the shock front in the form of the linear factor in Equation (33). The compositional diffusion is also subject to the time step limitation imposed in Equation (8). It is worth noting that compositional diffusion implies a flux of nuclear energy given by

$$
F_{\text {nuc }, \text { mix }}=\sum_{i} \frac{E_{\mathrm{B}, i} \mathcal{F}_{\mathrm{mix}, i}}{A_{i} m_{p}}
$$

The entropy transport equation implied by our algorithm is

$$
\rho T \frac{d s}{d t}+\frac{1}{r^{2}} \frac{\partial}{\partial r}\left(r^{2} F_{\mathrm{conv}, \mathrm{mod}}\right)=Q_{\mathrm{visc}}-Q_{\nu}+Q_{\mathrm{nuc}},
$$

where $d / d t=\partial / \partial t+v_{r} \partial / \partial r$ and

$$
Q_{\mathrm{nuc}}=-\rho \sum_{i} \frac{E_{\mathrm{B}, i}}{A_{i} m_{p}} \frac{\partial X_{i}}{\partial t}
$$

is the rate of heating or cooling associated with nuclear compositional transformation (see Equations (14) and (16)).

\subsection{Thin Disk Corrections}

We have thus far assumed a rotating, quasi-spherical accretion flow. However, near the black hole, where cooling by neutrino emission and nuclear photodisintegration into nucleons is significant, the flow can become geometrically thin (e.g., MacFadyen \& Woosley 1999; Popham et al. 1999; Kohri et al. 2005; Chen \& Beloborodov 2007). In this case, the quasispherical treatment underestimates the density, pressure, and temperature near the midplane of thin disk, where the bulk of the neutrino emission takes place. We introduce a correction that adjusts the temperature of the flow to be closer to the physical, thin-disk value. 
In what follows, the quantities applying to the thin disk will be marked with tilde. Let $\tilde{H}_{z}$ denote the vertical halfthickness of the thin disk. We assume that the vertical halfthickness of the quasi-spherical flow is $H_{z} \sim(1 / 2) r$. Since the thin and the quasi-spherical flow must contain the same column density, $\tilde{H}_{z} \tilde{\rho} \sim(1 / 2) r \rho$. Ignoring differences in nuclear composition between the thin and quasi-spherical flows, the same correspondence must apply to the total internal energies integrated along the vertical column, $\tilde{H}_{z} \tilde{\rho} \tilde{\epsilon} \sim(1 / 2) r \rho \epsilon$, and thus, $\tilde{\epsilon} \sim \epsilon$ while $\tilde{H}_{z} \tilde{P} \sim(1 / 2) r P$. Vertical force balance in the thin disk requires $\tilde{P} / \tilde{H}_{z}=\tilde{\rho}\left|g_{z}\right|$, where $\tilde{g}_{z}=-\operatorname{sgn}(z) g \tilde{H}_{z} / r$ is the gravitational acceleration in the $z$-direction and $g \equiv$ $\left(\mathbf{g}_{\mathrm{BH}}+\mathbf{g}_{\mathrm{self}}\right) \cdot \hat{\mathbf{r}}$ is the radial gravitational acceleration (see Section 2.2). Enforcing that $\tilde{H}_{z} \leqslant H_{z}$, we obtain

$$
\tilde{H}_{z}=\min \left[\left(-\frac{r P}{\rho g}\right)^{1 / 2}, \frac{r}{2}\right] .
$$

To account for the higher density in the thin disk, we could pass $\tilde{\rho}$ to the EOS. This, however, would result in a modified pressure $\tilde{P}$. Out of a possibly unfounded concern that a modification of the pressure would introduce spurious dynamics in the spherically averaged flow, we opted to modify our estimate of the disk midplane temperature in a manner not directly affecting the fluid pressure. This corrected temperature then enters the calculation of the neutrino cooling rate and the NSE composition, both of which are highly sensitive to the midplane temperature.

We estimate the midplane temperature $\tilde{T}$ from the following extrapolation:

$$
\ln \left(\frac{\tilde{T}}{T}\right) \approx\left(\frac{\partial \ln T}{\partial \ln \rho}\right)_{\epsilon} \ln \left(\frac{\tilde{\rho}}{\rho}\right),
$$

where the partial derivative, which we denote with $\chi$, is evaluated at constant specific internal energy and can be expressed as

$$
\begin{aligned}
\chi & \equiv\left(\frac{\partial \ln T}{\partial \ln \rho}\right)_{\epsilon} \\
& =-\left(\frac{\partial s}{\partial \ln \rho}\right)_{T} /\left(\frac{\partial s}{\partial \ln T}\right)_{\rho}-\frac{P}{\rho c_{V} T},
\end{aligned}
$$

where $c_{V}$ is the specific heat at constant volume. The quantities on the right-hand side of Equation (42) are all provided by the Helmholtz EOS.

Since $\tilde{\rho} / \rho \sim r /\left(2 \tilde{H}_{z}\right)$, the midplane temperature of the disk can be approximated via

$$
\tilde{T}=\left(\frac{r}{2 \tilde{H}_{z}}\right)^{\chi} T \equiv \Xi T,
$$

where the last equality defines the dimensionless temperature correction factor $\Xi$. To ensure continuity near the shock transition, we modify the correction factor to linearly approach unity at the shock transition by defining

$$
\Xi_{\text {mod }}=1+\left(1-\frac{r}{r_{\text {shock }}}\right)(\Xi-1) .
$$

For clarity of notation, we drop the subscript in $\Xi_{\text {mod }}$ in what follows.
The correction introduced in Equation (43) affects both the temperature calculated from internal energy via the EOS and the NSE temperature calculated from the total internal and nuclear energy as described in Section 2.3 above. Equation (13) is corrected to become

$$
\epsilon_{\mathrm{EOS}}\left(\rho, \Xi^{-1} \tilde{T}, \mathbf{X}\right)=\epsilon,
$$

while Equation (17) is corrected to become

$$
\begin{aligned}
& \epsilon_{\mathrm{EOS}}\left[\rho, \Xi^{-1} \tilde{T}_{\mathrm{NSE}}, \mathbf{X}_{\mathrm{NSE}}\left(\tilde{\rho}, \tilde{T}_{\mathrm{NSE}}, Y_{e}\right)\right] \\
& \quad+\epsilon_{\mathrm{nuc}}\left[\mathbf{X}_{\mathrm{NSE}}\left(\tilde{\rho}, \tilde{T}_{\mathrm{NSE}}, Y_{e}\right)\right] \\
& \quad=\epsilon\left(\rho, \Xi^{-1} \tilde{T}, \mathbf{X}\right)+\epsilon_{\mathrm{nuc}}(\mathbf{X}) .
\end{aligned}
$$

Note that since $\Xi \geqslant 1$, the estimated midplane disk temperature is higher than the temperature calculated without this correction, but this allows the disk to cool faster than it would otherwise. The rate of cooling by neutrino emission is then calculated from Equations (20) and (21) but at density $\tilde{\rho}$ and temperature $\tilde{T}$.

\subsection{Initial Model and Boundary Conditions}

The initial model is the rotating $M_{\text {star }} \approx 14 M_{\odot}$ W-R star $16 \mathrm{TI}$ of Woosley \& Heger (2006), evolved to pre-core-collapse from a $16 M_{\odot}$ main-sequence progenitor. ${ }^{3}$ To prepare the model 16TI, Woosley \& Heger assumed that the rapidly rotating progenitor, which is near breakup at its surface at $r_{\text {star }} \approx 4 \times 10^{5} \mathrm{~km}$, had low initial metallicity, $0.01 Z_{\odot}$, and became a W-R star shortly after central hydrogen depletion, which implied an unusually small amount of mass loss. For illustration, the specific angular momentum at the three-quarters mass radius was $\ell_{3 / 4} \sim 8 \times 10^{17} \mathrm{~cm}^{2} \mathrm{~s}^{-1}$, implying circularization around a $5 M_{\odot}$ black hole at $r \sim 2500 \mathrm{~km}$, much larger than ISCO. The circularization radii of the outermost layers of the star are in the range $10^{4}-10^{5} \mathrm{~km}$. Woosley \& Heger provide a radiusdependent angular momentum profile $\ell_{16 \mathrm{TI}}(r)$. We introduce the dimensionless parameter $\xi_{\ell}$ to scale the specific angular momentum $\ell(r)$ of our initial model relative to that of $16 \mathrm{TI}$

$$
\ell(r)=\xi_{\ell} \ell_{16 \mathrm{TI}}(r) .
$$

The plots of density, temperature, angular momentum, and composition in Section 3 show the initial conditions. The angular momentum profile is specific to our fiducial Run 1 with $\xi_{\ell}=0.5$, half of the rotation rate of $16 \mathrm{TI}$.

The iron core of the model 16TI, with a mass $\sim 1 M_{\odot}$, has mass too low to collapse directly into a black hole, but should instead first collapse into a neutron star. The latter could, but need not, be driven to a successful explosion by the delayed neutrino mechanism. A black hole can form by fallback. We do not in any way account for the core bounce and its consequences, nor for the heating by the neutrinos emitted from the proto neutron star. Our central compact object is a point mass from the outset equipped with, as we clarify below, an absorbing boundary condition.

Pseudo-logarithmic gridding is achieved by capping the adaptive resolution at radius $r$ with $\Delta r>(1 / 8) \eta r$ where $\eta$ is a dimensionless parameter. We choose $\eta=0.15$ for all but Run 2, where $\eta=0.075$. Beyond the outer edge of the star

\footnotetext{
3 López-Cámara et al. (2009) carried out SPH simulations of neutrino-cooled accretion during the first $0.5 \mathrm{~s}$ of the collapse and Morsony et al. (2007) and Nagakura et al. (2011a) simulated the propagation of a relativistic jet using the same model star. We discuss important caveats of using this model in Section 4.
} 
Table 1

Summary of Simulation Parameters

\begin{tabular}{lrlllr}
\hline \hline Run & $\begin{array}{c}\Delta r_{\text {min }} \\
(\mathrm{km})^{\mathrm{a}}\end{array}$ & $\alpha^{\mathrm{b}}$ & $\xi_{\ell} \ell^{\mathrm{c}}$ & $\xi_{\text {conv }}{ }^{\mathrm{d}}$ & $\xi_{\text {conv, mix }} \mathrm{e}$ \\
\hline 1 & 10.0 & 0.1 & 0.5 & 2.0 & 6.0 \\
$2^{\mathrm{f}}$ & $\mathbf{0 . 5}$ & 0.1 & 0.5 & 2.0 & 6.0 \\
3 & 10.0 & $\mathbf{0 . 2}$ & 0.5 & 2.0 & 6.0 \\
4 & 10.0 & $\mathbf{0 . 0 2 5}$ & 0.5 & 2.0 & 6.0 \\
5 & 10.0 & 0.1 & 0.5 & $\mathbf{5 . 0}$ & $\mathbf{1 5 . 0}$ \\
6 & 10.0 & 0.1 & 0.5 & $\mathbf{0 . 5}$ & 6.0 \\
7 & 10.0 & 0.1 & 0.5 & $\mathbf{1 . 0}$ & 6.0 \\
8 & 10.0 & 0.1 & $\mathbf{0 . 2 5}$ & 2.0 & 6.0 \\
9 & 10.0 & 0.1 & 0.5 & 2.0 & $\mathbf{3 . 0}$ \\
\hline
\end{tabular}

Notes.

a The minimum resolution element size.

b The dimensionless viscous stress-to-pressure ratio.

c Rotational profile parameter (see Equation (47)).

${ }^{\mathrm{d}}$ Convective efficiency parameter (see Equation (27)).

e Convective compositional mixing efficiency parameter (see Equation (34)).

f This run also had additional angular resolution (see Section 2.7).

we place a cold $\left(10^{4} \mathrm{~K}\right)$, low-density, stellar-wind-like medium with density profile $\rho(r)=3 \times 10^{-7}\left(r / r_{\text {star }}\right)^{-2} \mathrm{~g} \mathrm{~cm}^{-3}$.

The simulation was carried out in the spherical domain $r_{\min }<r<r_{\max }$. We placed the inner boundary at $r_{\min } \sim 25 \mathrm{~km}$ and the outer boundary well outside the star at $r_{\max }=10^{7} \mathrm{~km}$. In Table 1, we summarize the main parameters of our simulations, and also present some of the key measurements, defined in Section 3, characterizing the outcome of each simulation. Each simulation was run for $\sim 10^{7}$ hydrodynamic time steps and required $\sim 5000 \mathrm{CPU}$ hours to complete.

The boundary condition at $r_{\min }$ was unidirectional "outflow" that allowed free flow from larger to smaller radii $\left(v_{r}<0\right)$ and disallowed flow from smaller to larger radii $\left(v_{r}>0\right)$ by imposing a reflecting boundary condition. We imposed the torque-free boundary condition via (see, e.g., Zimmerman et al. 2005)

$$
\frac{\partial}{\partial r}\left(\frac{\ell}{r^{2}}\right)_{r=r_{\min }}=0 .
$$

As in other Eulerian codes, the boundary conditions in FLASH are set by assigning values to fluid variables in rows of "guard" cells just outside the boundary of the simulated domain. Let $r_{1 / 2}$ denote the leftmost cell within the simulated domain, and let $r_{\mathcal{G}}$ where $\mathcal{G}=(-7 / 2,-5 / 2,-3 / 2,-1 / 2)$ be the four guard cells to the left of $r_{1 / 2}$ such that the grid separation corresponds to $\Delta \mathcal{G}=1$. The torque-free boundary condition, if assumed to apply for $r \leqslant r_{\min }$, implies $\ell_{\mathcal{G}} / r_{\mathcal{G}}^{2}=\ell_{1 / 2} / r_{1 / 2}^{2}$. All other fluid variables $X$ were simply copied into the guard cells, $X_{\mathcal{G}}=X_{1 / 2}$, and were subsequently rendered thermodynamically consistent. This simple prescription approximates free inflow (toward smaller $r$ ) across $r_{\text {min }}$. The guard cell values for other fluid variables are assigned ignoring curvature of the coordinate mesh and formally violate conservation laws at $r<r_{\min }$.

The mass of the black hole $M_{\mathrm{BH}}$ was initialized with the mass of the initial stellar model contained within $r_{\text {min }}$. The black hole mass was evolved by integrating the mass crossing the boundary at $r=r_{\min }$,

$$
\frac{d M_{\mathrm{BH}}}{d t}=\left(-4 \pi r^{2} \rho v_{r}\right)_{r=r_{\min }} .
$$

The sum of the mass of the black hole and the mass contained on the computational grid remains constant to a high level of precision throughout each simulation.

\subsection{Assessment and Tests of the Code}

We conducted tests of internal energy conservation, angular momentum transport, and spatial resolution convergence. The time-integrated equation for the conservation of internal energy in absence of nuclear and thermal energy interconversion in spherical coordinates reads

$$
\begin{gathered}
E_{\text {int }, \text { tot }}\left(t_{\max }\right)-E_{\text {int, tot }}\left(t_{\min }\right)=4 \pi r_{\text {min,test }}^{2} \int_{t_{\min }}^{t_{\max }}\left(v_{r} \rho \epsilon+F_{\text {conv }}\right) d t \\
-4 \pi \int_{t_{\min }}^{t_{\max }} \int_{r_{\text {min,test }}}^{r_{\max }}\left[\frac{P}{r^{2}} \frac{\partial\left(r^{2} v_{r}\right)}{\partial r}-Q_{\mathrm{visc}}+Q_{v}\right] r^{2} d r d t=0,
\end{gathered}
$$

where

$$
E_{\text {int,tot }}=\int_{r_{\text {min,test }}}^{r_{\max }} 4 \pi r^{2} \rho \epsilon d r
$$

and $r_{\text {min,test }} \geqslant r_{\min }$ is a reference radius defining the inner boundary of the spherical annulus in which we test energy conservation. We have ignored any flow of energy through $r_{\max }$, since stellar material does not reach this radius in the course of any simulation.

In Figure 1, we utilize Equation (50) to test the global conservation of internal energy in a run with identical test parameters to Run 2, except that we disabled nuclear compositional change and used a Newtonian gravitational potential without relativistic corrections. In the legend, the apparent error $\Delta E$ is defined as the absolute value of the difference between the left- and righthand sides of Equation (50). The evaluation of the various terms in Equation (50) was carried out in post-processing from cellcentered data recorded in $\Delta t=0.01 \mathrm{~s}$ intervals, which, in retrospect, is prone to the introduction of various spatial and temporal discretization artifacts not present in the actual simulation.

We find that the apparent error is $<1 \%$ of the largest term in Equation (50) when calculated for $r_{\text {min,test }}=50 \mathrm{~km}$ for the time interval $0 \mathrm{~s} \leqslant t \leqslant 70 \mathrm{~s}$. The apparent error is most significant, $\Delta E \sim 4 \times 10^{50} \mathrm{erg}$, prior to and during the first few seconds after shock passage. The apparent error that accrues after the first few seconds following shock passage is less than $10^{50} \mathrm{erg}$. This can be compared to the total binding energy change on the simulation grid, which if sufficiently large can imply a supernova. We calculate this energy in Section 3.5 below and find that it increases by $\sim(1.5-2) \times 10^{51} \mathrm{erg}$ following shock formation and a significant fraction $\left(\sim 5 \times 10^{50} \mathrm{erg}\right)$ of the increase is accrued later than a few seconds after shock formation, when the change in the cumulative apparent error is very small. Therefore, it does not seem that the apparent energy conservation error at the levels seen in the simulations should significantly impact the prospects for explosion. We note that in our calculations we explicitly transport specific internal energy rather than the total energy, by setting the parameter eintSwitch to a very large value. We would like to reiterate that it is likely that the apparent error is an artifact of post-processing and the true energy conservation is better. To demonstrate the latter, however, one would have to reconstruct the diagnostic energy fluxes using the very same interpolation procedure as is performed within the PPM in FLASH. We anticipate carrying out such a test in an extension of this work.

In steady state accretion, mass accretion associated with viscous angular momentum transport should occur at the rate

$$
\dot{M}_{\text {s.s. }}=-4 \pi\left(\frac{\partial \ell}{\partial r}\right)^{-1} \frac{\partial}{\partial r}\left(r^{4} v \rho \frac{\partial \Omega}{\partial r}\right) .
$$



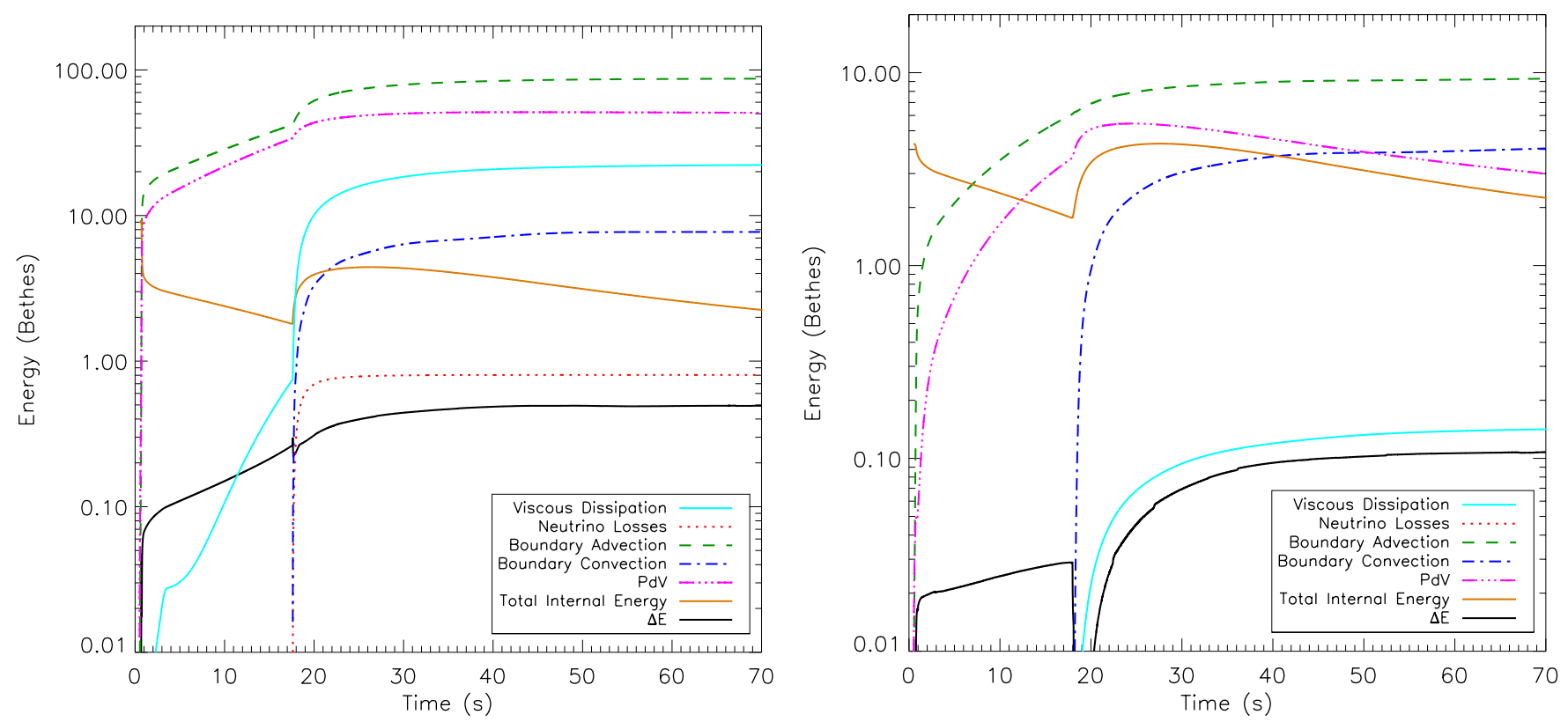

Figure 1. Test of internal energy conservation in a run identical to Run 2 except with nuclear compositional change disabled and using a Newtonian gravitational potential. Plotted are each of the terms in Equation (50) calculated at $r_{\min }=50 \mathrm{~km}$ (left) and $r_{\min }=1000 \mathrm{~km}$ (right). Note the difference in scales on the vertical axis. The quantity $\Delta E$ represents the absolute value of the difference between the left- and right-hand sides of Equation (50). A possibly dominant source of apparent error is inconsistent discretization of the various fluid variables and fluxes in PPM and in the post-processing and need not reflect an inaccuracy of the computation. Neutrino losses are insignificant outside the inner $\sim 1000 \mathrm{~km}$. Over the interval $0 \mathrm{~s} \leqslant t \leqslant 70 \mathrm{~s}$, we find that the total error is $<1 \%$ of the largest term in Equation (50). (A color version of this figure is available in the online journal.)

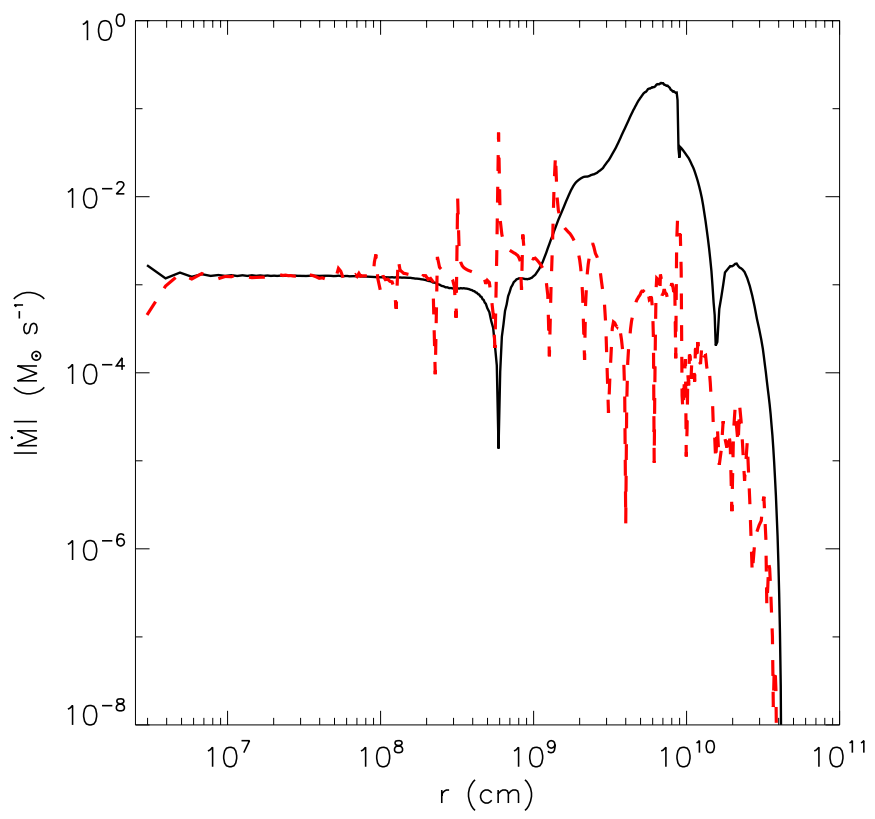

Figure 2. Absolute value of the actual (black, solid) and steady state analytic (red, dotted, see Equation (52)) mass flow, $\dot{M}$, as a function of radius in Run 1 at $t=50 \mathrm{~s}$. The deviation from the analytical value is $\lesssim 5 \%$ at radii $100 \mathrm{~km} \lesssim r \lesssim 1000 \mathrm{~km}$. At this time, $r_{\text {shock }} \sim 7 \times 10^{4} \mathrm{~km}$.

(A color version of this figure is available in the online journal.)

In Figure 2 we show $\dot{M}(r)$ for Run 2 at $t=50 \mathrm{~s}$ along with the analytic steady-state estimate of Equation (52). In the rotationally supported region $100 \mathrm{~km} \lesssim r \lesssim 1000 \mathrm{~km}$, the deviation from the analytical value is $\lesssim 5 \%$, which lends credence to the accuracy of our angular momentum transport scheme.

Our spatial resolution was chosen such that we resolve the innermost, neutrino-cooled region of the disk over several zones.
One caveat is that we do not resolve the sonic radius of the flow, an issue discussed in McKinney \& Gammie (2002). Because we use a torque-free boundary condition, the calculation is not subject to spurious viscous dissipation at the inner boundary. However, our boundary condition may still influence the fluid flow, and it therefore may be more apt to consider values of $\dot{M}$, as opposed to, for example, $\alpha$ or $\ell$, when comparing our work to other simulations.

Run 1 and Run 2 contained identical hydrodynamic parameters and differed only in spatial resolution. Run 2 was capable of one additional level of resolution refinement over Run 1 and the parameter $\eta$ described in Section 2.7 was set to one-half the value in Run 1, allowing for significantly higher resolution as a function of radius. Figures 3-6 show the density, temperature, specific entropy, and the mean atomic weight in Run 1, and also in the higher-resolution Run 2 at different times. Substantial agreement is seen between the low- and high-resolution simulations.

\subsection{Limitations of the Method}

The primary limitations of the model of collapsar accretion that we have presented here include: (1) a very approximate one-dimensional treatment of the intrinsically two- and three-dimensional flow structures; (2) limited adequacy of the Navier-Stokes viscous stress as a model for the magnetic stress arising in the nonlinear development of the MRI; (3) no a priori knowledge of the expected efficiency of convective energy transport $\xi_{\text {conv }}$ and of compositional transport $\xi_{\text {conv, mix }}$; (4) treatment of nuclear compositional transformation through relaxation to NSE rather than by integrating the nuclear reaction network that would have allowed us to make predictions about the nucleosynthetic output; (5) neglect of ambient compositional stratification and nuclear compositional transformation inside convective cells in the calculation of the convective heat flux; (6) the lack of modeling of the axial relativistic jet and 

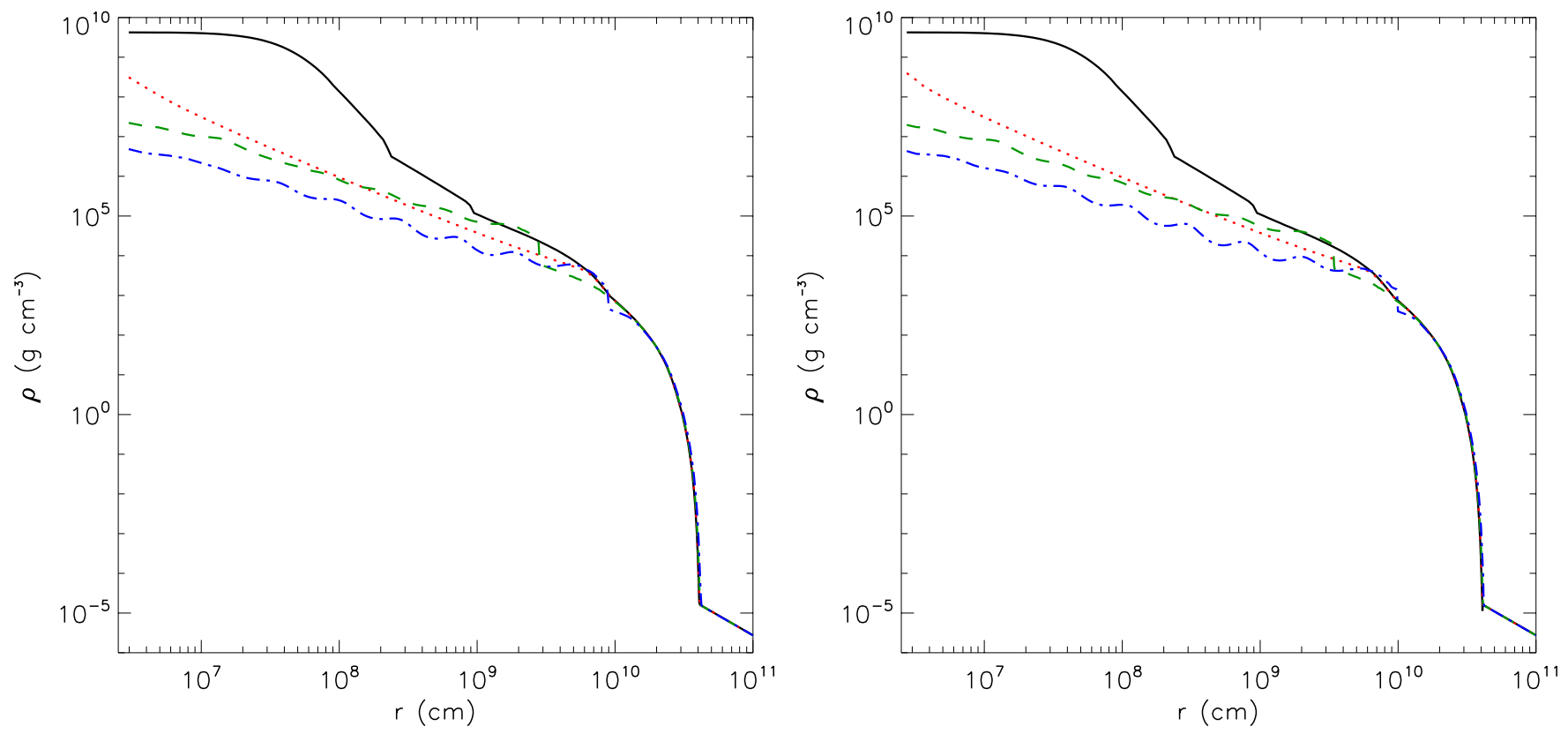

Figure 3. Density in Run 1 (left) and the higher resolution Run 2 (right) at $t=0 \mathrm{~s} \mathrm{(black,} \mathrm{solid),} t=15 \mathrm{~s}$ (red, dotted), $t=25 \mathrm{~s}$ (green, dashed), and $t=50 \mathrm{~s} \mathrm{(blue,}$ dash-dotted). In the convective region behind the shock front, some waves form due to an instability, developing at late times, which is likely an artifact of including mixing length theory convection as an explicit term in the transport equations. The density jump across the shock front is approximately an order of magnitude. (A color version of this figure is available in the online journal.)
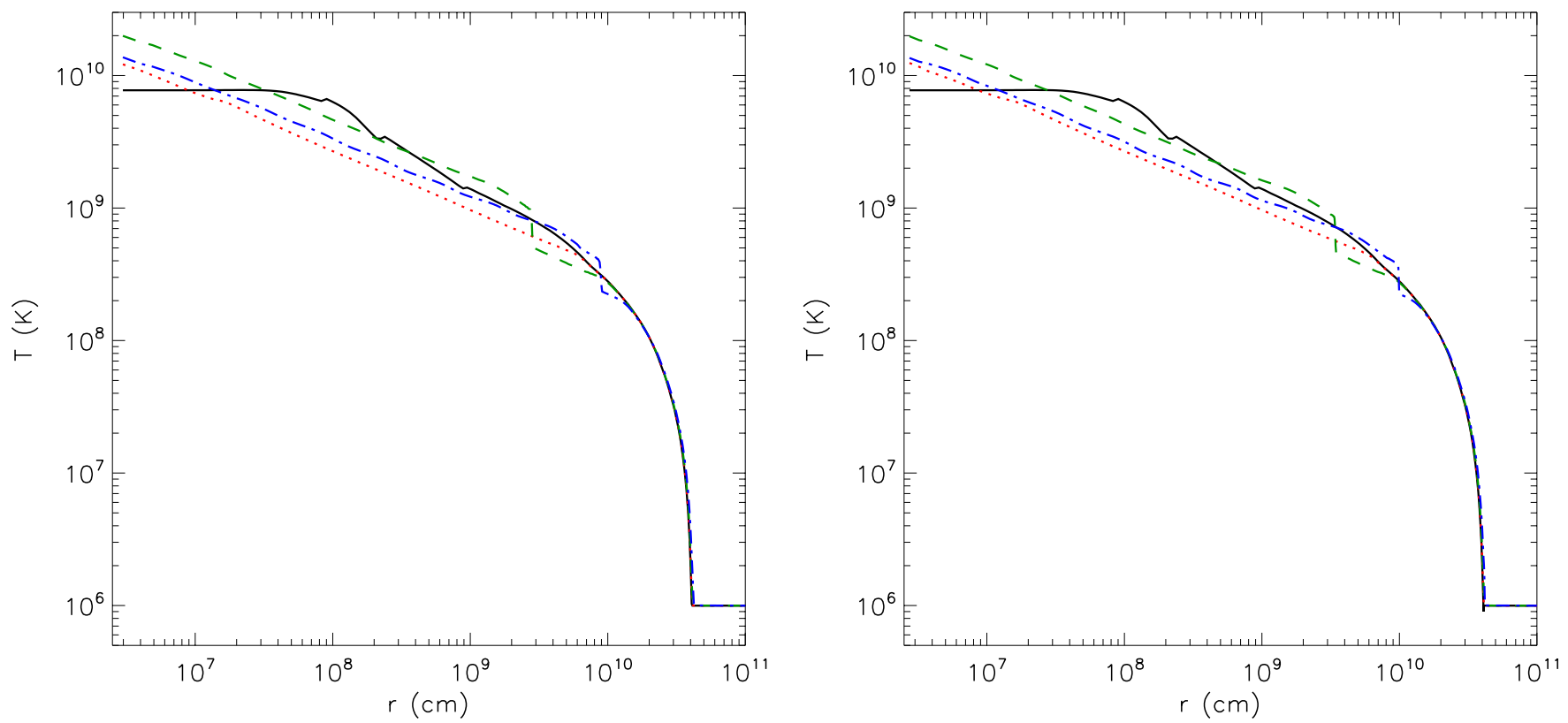

Figure 4. Temperature in Run 1 (left) and the higher resolution Run 2 (right) at $t=0 \mathrm{~s}$ (black, solid), $t=15 \mathrm{~s}$ (red, dotted), $t=25 \mathrm{~s}$ (green, dashed), and $t=50 \mathrm{~s}$ (blue, dash-dotted). Photodisintegration and neutrino emission cool the innermost disk, while nuclear fusion provides additional heating in the post-shock region (see Section 3.4).

(A color version of this figure is available in the online journal.)

its enveloping cocoon that are thought to be present in LRGB sources; and (7) the use of a relatively low mass progenitor star, which may or may not be able to yield a black hole and an explosion with an energy as high as has been inferred in supernovae associated with LGRBs. Overcoming limitations (1) through (6) will require much more computationally expensive multidimensional hydrodynamic and MHD simulations. Limitation (7) can be addressed by applying our current method to other, more massive stellar models; here, we speculate what collapsars in higher mass progenitors may behave like in Section 4 below.
It would be tempting in view of limitation (5) to try to incorporate the effects of compositional stratification ambient to convective cells in the convective energy flux, which is normally achieved by multiplying the energy flux in Equation (27) with a factor

$$
\left[1-\left(\frac{d T}{d \mu}\right)_{P, \rho} \frac{d \mu}{d r} /\left(\frac{T}{c_{p}} \frac{d s}{d r}\right)\right]^{1 / 2},
$$

where $\mu$ is the mean nuclear mass, and utilizing the Ledoux instead of the Schwarzschild criterion (see, e.g., Bisnovatyi-Kogan 

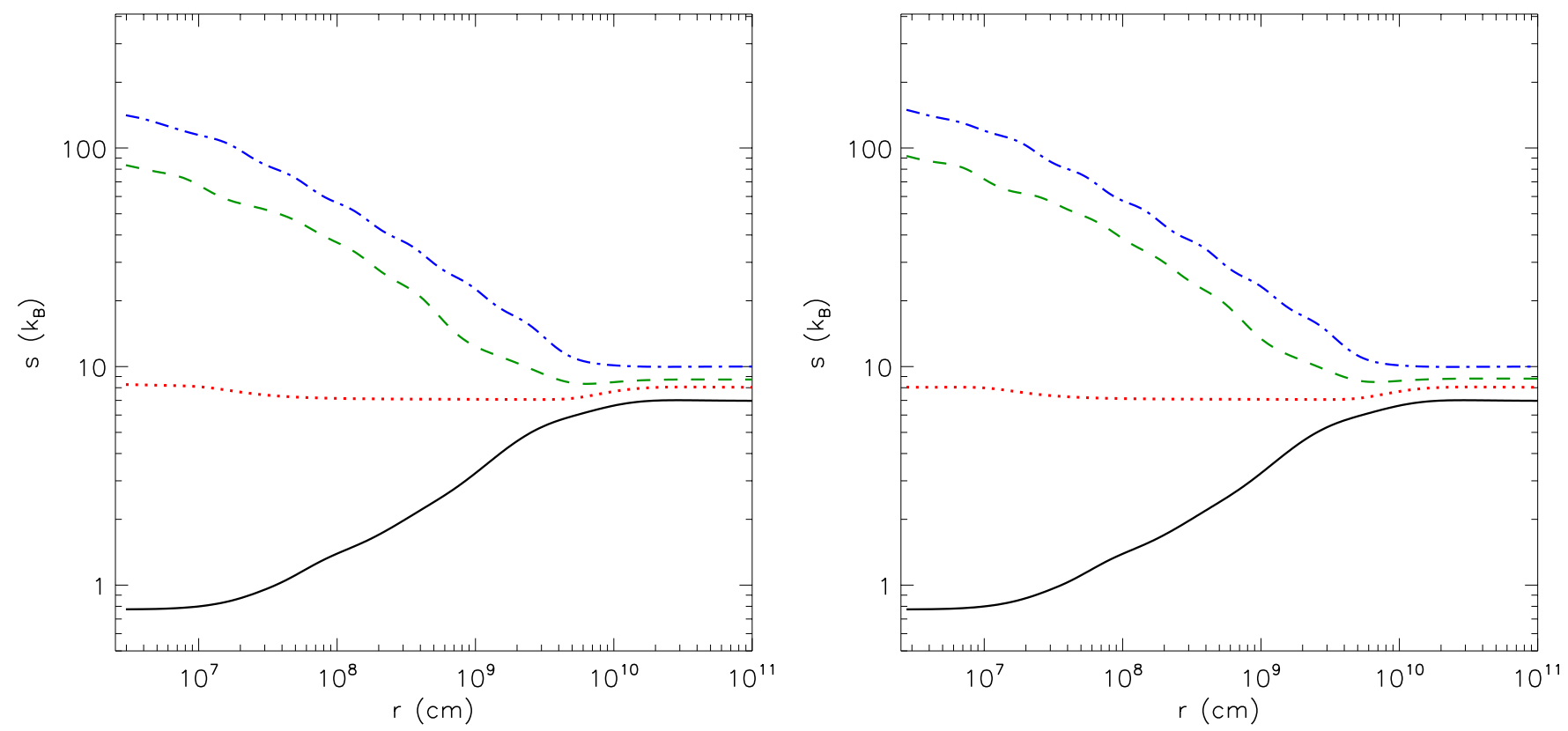

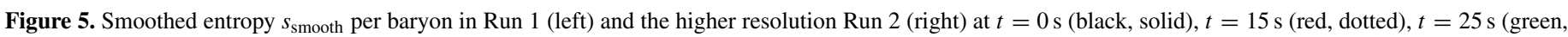
dashed), and $t=50 \mathrm{~s}$ (blue, dash-dotted) in units of the Boltzmann constant $\left(k_{\mathrm{B}}\right)$. After fluid comes into radial force balance, a strong entropy inversion is observed, giving rise to convection.

(A color version of this figure is available in the online journal.)
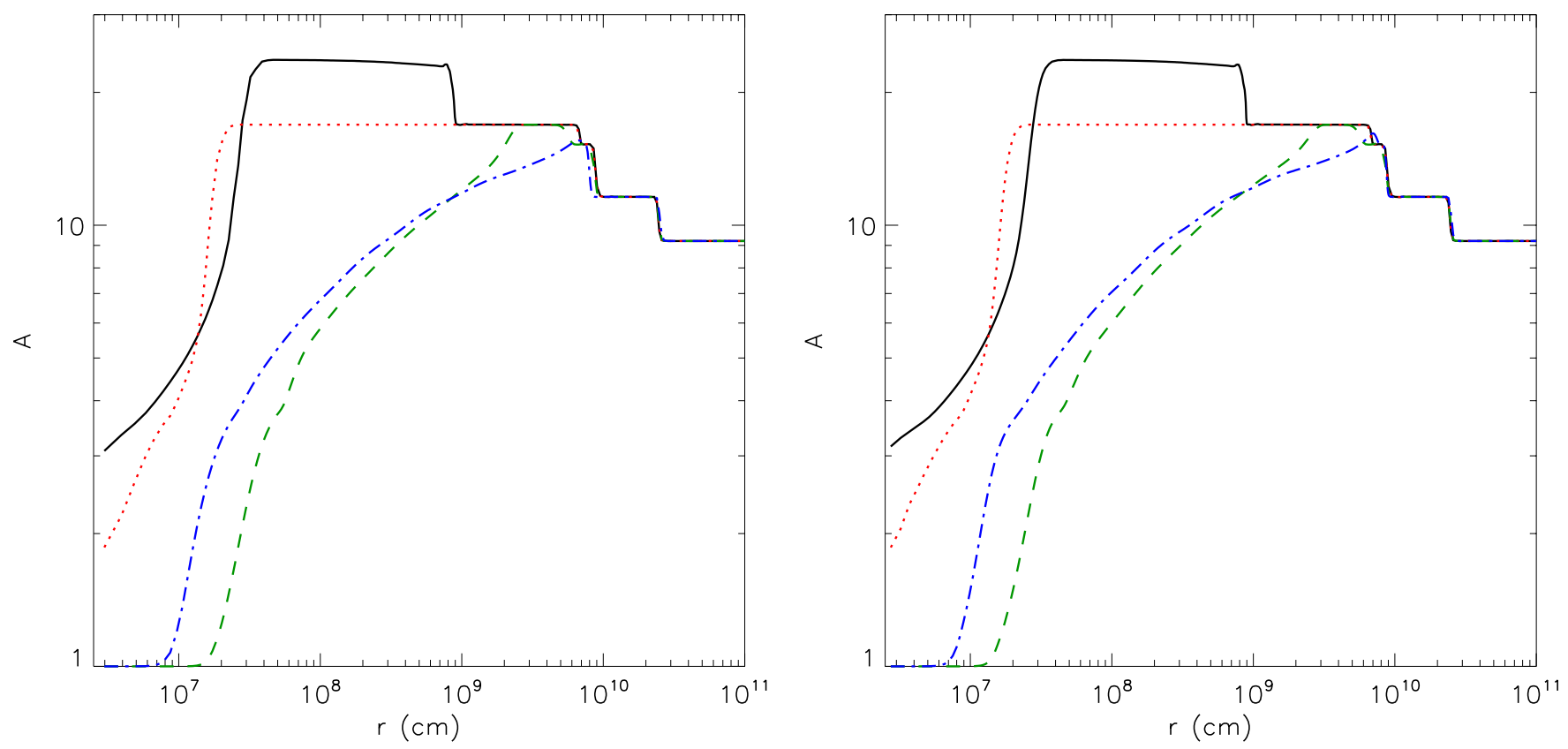

Figure 6. Mass-weighted average of the atomic mass $\bar{A}$ in Run 1 (left) and Run 2 (right) at $t=1 \mathrm{~s}$ (black, solid), $t=15 \mathrm{~s}$ (red, dotted), $t=25 \mathrm{~s}$ (green, dashed), and $t=50 \mathrm{~s}$ (blue, dot-dashed). Note that at $t=1 \mathrm{~s}$, the iron core has already accreted onto the central point mass. At late times, photodisintegration in the hottest inner regions behind the shock front reduces the value of $\bar{A}$. Convective mixing is able to dredge up lighter elements; our scheme for nuclear compositional transformation does not correctly model the subsequent recombination and freezeout well outside NSE.

(A color version of this figure is available in the online journal.)

2001). This would be meaningful as long as the convective eddy turnover time $\tau_{\text {conv }} \sim \lambda_{\text {conv }} / v_{\text {conv }}$ were shorter than the nuclear timescale $\tau_{\text {nuc }} \lesssim \tau_{\mathrm{NSE}}$, so that the convective cells can be treated as adiabatic before they mix. However, at radii where Ledoux convection would differ most from Schwarzschild convection, namely, where the photodissociation into helium nuclei and free nucleons is substantial, the convective timescale is much longer than the nuclear timescale, $\tau_{\text {conv }} \gg \tau_{\text {nuc }}$. The internal composition of a convective cell evolves as it rises, and the associated entropy change is a much stronger effect than the variation of the ambient composition treated in Ledoux convection. Magnetization of the medium may play a role in this regime but its effects are poorly understood. Research into the interplay of convection and nuclear burning is ongoing (see, e.g., Arnett \& Meakin 
2011). Cognizant of these and other limitations, we adopt the Schwarzschild model and consider it but a parameterization of complex, still-to-be-explored physics.

\section{RESULTS}

Nine simulations were carried out to explore sensitivity to the resolution of the simulation $\Delta r_{\text {min }}$, the viscous stress-to-pressure ratio $\alpha$, the stellar rotation $\xi_{\ell}$, the efficiency of convective energy transport $\xi_{\text {conv }}$, and the efficiency of convective mixing $\xi_{\text {conv,mix }}$. The values of these parameters in each of the simulations are summarized in Table 1. Among these, Run 1 can be considered the fiducial model. Each simulation was run for $100 \mathrm{~s}$, except for Runs 4 and 5, where strong numerical instabilities associated with our convection scheme prevented us from simulating for more than $40 \mathrm{~s}$ and $50 \mathrm{~s}$, respectively. In what follows, we present the results. In Section 3.1, we address the evolution of the rate with which mass accretes onto the central black hole. In Section 3.2, we discuss the nature of radial force balance in the fraction of the stellar material that has been traversed by the outgoing shock wave, but has not accreted onto the black hole and also discuss the mass and angular momentum transport in the system. In Section 3.3, we address energy transport. In Section 3.4, we address the nuclear composition of the flow and discuss the limitations inherent in our simplified treatment of nuclear compositional transformation. In Section 3.5, we discuss the global energetics and check whether sufficient energy may be transported into a portion of the stellar envelope to produce a supernova.

\subsection{Central Accretion Rate and Black Hole Mass}

Each simulation exhibits unshocked radial accretion of the inner, low-angular momentum mass shells of the progenitor star through the inner boundary lasting $\sim(20-30)$ s at relatively steady accretion rates of $\sim(0.1-0.2) M_{\odot} \mathrm{s}^{-1}$. The central mass accretion rate, black hole mass, and total mass on the computational grid as a function of time in each simulation are shown in Figure 7. The abrupt drop of the central accretion rate at $\sim(20-30) \mathrm{s}$ is associated with the appearance of an accretion shock precipitated by the arrival of the mass shells with specific angular momentum sufficient to lead to circularization around the black hole.

In Figure 8, we show the location of the shock $r_{\text {shock }}$ and its velocity $v_{\text {shock }} \equiv d r_{\text {shock }} / d t$ as a function of time. For each of the runs, we identify the time when the shock first reaches radius $10 r_{\min }=250 \mathrm{~km}$ as the shock formation time $t_{\text {shock }}$ and list the shock formation times in Column 2 of Table 2. We also provide the mass of the black hole at this point, $M_{\mathrm{BH}}\left(t_{\text {shock }}\right)$, in Column 8. The black hole mass at the time of shock formation was $M_{\mathrm{BH}}\left(t_{\text {shock }}\right) \sim(5.2-5.5) M_{\odot}$ in Runs 1-7 and 9. In Run 8, which was initiated with reduced initial angular momentum, the accretion shock appeared later and the black hole mass is correspondingly larger.

After the formation of the shock, the fluid nearest the inner boundary is rotationally supported and accretes as a result of angular momentum transport driven by the viscous shear stress. Subsequent to shock formation, the accretion rate declines rapidly either promptly or following a short delay. The typical rapid drop of the accretion rate is by a factor $\sim 5-10$ (Runs 1 , $2,3,5,7$, and 9), and this is followed by a continued powerlaw-like decline. By the end of each simulation at $100 \mathrm{~s}$, the accretion rate has typically declined to $\sim\left(10^{-3}-10^{-4}\right) M_{\odot} \mathrm{s}^{-1}$ (Runs 1, 2, 3, 4, 7, 8, and 9) or a factor of 100-1000 of the
Table 2

Summary of Key Measurements

\begin{tabular}{lcccccc}
\hline \hline Run & $t_{\text {shock }}{ }^{\mathrm{a}}$ & $M_{\mathrm{BH}}\left(t_{\text {shock }}\right)^{\mathrm{b}}$ & $M_{\text {unbound }}{ }^{\mathrm{c}}$ & $E_{\text {bind }}{ }^{\mathrm{d}}$ & $E_{\text {kin }}{ }^{\mathrm{e}}$ & $M_{\mathrm{Fe}}{ }^{\mathrm{f}}$ \\
\hline 1 & 20.3 & 5.4 & 6.0 & 0.40 & 0.31 & 0.06 \\
$2^{\mathrm{g}}$ & 19.1 & 5.2 & 6.4 & 0.44 & 0.36 & 0.04 \\
3 & 19.2 & 5.2 & 5.7 & 0.34 & 0.28 & 0.06 \\
4 & 19.8 & 5.3 & 4.4 & 0.62 & 0.29 & 0.07 \\
5 & 20.6 & 5.5 & 3.1 & 0.40 & 0.18 & 0.04 \\
6 & 20.3 & 5.4 & 0.0 & -0.43 & 0.16 & 0.03 \\
7 & 19.2 & 5.2 & 4.4 & 0.08 & 0.17 & 0.09 \\
8 & 34.0 & 7.9 & 5.9 & 0.54 & 0.29 & 0.02 \\
9 & 19.2 & 5.2 & 6.8 & 0.46 & 0.37 & 0.03 \\
\hline
\end{tabular}

Notes.

a Time at which shock reaches $r=250 \mathrm{~km}(\mathrm{~s})$.

b Black hole mass at when the shock reaches $r=250 \mathrm{kms}\left(M_{\odot}\right)$.

${ }^{\mathrm{c}}$ Unbound mass at the end of the simulation $\left(M_{\odot}\right)$.

${ }^{\mathrm{d}}$ Total energy in the stellar material at the end of the simulation $\left(10^{51} \mathrm{erg} \mathrm{s}^{-1}\right.$; see Section 3.5 and Figure 18).

e Total kinetic energy of outbound material $\left(10^{51} \mathrm{erg} \mathrm{s}^{-1}\right.$; see Section 3.5).

$\mathrm{f}$ Total mass of newly synthesized Fe-group elements at the end of the simulation ( $M_{\odot}$; see Section 3.4).

$\mathrm{g}$ This run also had additional angular resolution (see Section 2.7).

pre-shock value. Final black hole masses were $\sim(6-7) M_{\odot}$ in the simulations with $\xi_{\ell}=0.5$ and $\sim 10 M_{\odot}$ in Run 8 with reduced initial angular momentum $\xi_{\ell}=0.25$.

In Run 4, with a low value of the viscosity parameter $\alpha=$ 0.025 , the shock first made a very slow progress from $300 \mathrm{~km}$ to $2000 \mathrm{~km}$ during the first $10 \mathrm{~s}$ from its appearance. Then, at $30 \mathrm{~s}$, the shock suddenly accelerated to $v_{\text {shock }} \sim 5000 \mathrm{kms}^{-1}$. The near-stagnation of the shock can be understood by noticing that during the $10 \mathrm{~s}$, the neutrino cooling rate matches the viscous heating rate; the rapid cooling prevents the central entropy rise and convection seen in all other runs (see Section 3.3 below). In Section 4.2 of Lindner et al. (2010), we discussed the scenario in which the shock stagnation brought about by efficient neutrino cooling prolongs the LGRB central engine activity resulting in a longer prompt emission.

In Run 6, which had convective efficiency $\xi_{\text {conv }}=0.5$, the shock stalled at the radius $\sim 10^{4} \mathrm{~km}$ for $\sim 5 \mathrm{~s}$ before proceeding outward. Note that the reinvigoration of the shock is solely driven by the convective energy transport, as we do not simulate the negligible neutrino energy and momentum deposition. The stalling and restarting of the shock was reflected in a strong variability of the central accretion rate.

\subsection{The Shocked Envelope and Angular Momentum}

Shock passage leaves a shock- and convection-heated, pressure supported envelope which contains much more mass than the disk, consistent with what we saw in Lindner et al. (2010). Figure 3 shows that the density in the envelope is an approximate power law of radius $\rho \propto r^{-0.9}$. Figure 4 indicates that the temperature is also a power-law $T \propto r^{-0.4}$. The pressure (not shown) is an approximate power law $P \propto r^{-1.8}$. The profiles extend inward into the regime in which rotational support dominates pressure support. The mass of the rotationally supported material in the grid, where $a_{\text {cent }}>(1 / 2)\left|g_{\text {self }}+g_{\text {BH }}\right|$, promptly following disk formation was typically $\lesssim 5 \%$ of the total mass on the grid. Most of the mass on the grid was in the pressure supported atmosphere seamlessly connecting to the disk. The mass of the disk in each simulation is shown in Figure 7. In some of the runs, certain variability is seen in the disk mass over the first few seconds of disk formation. Afterward, the disk mass in each 

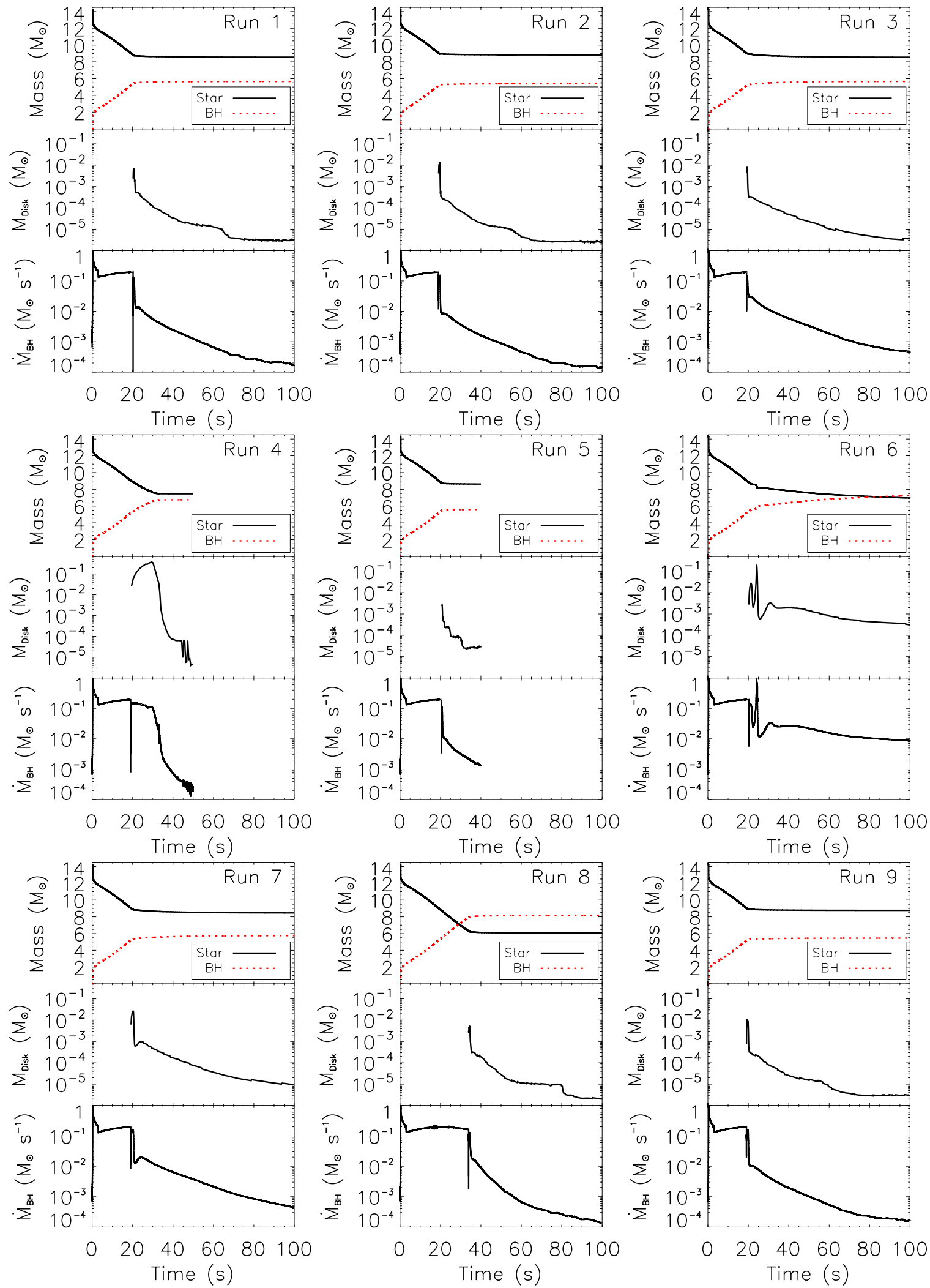

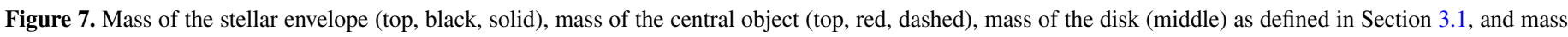

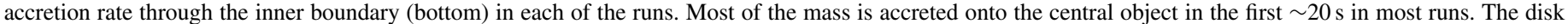

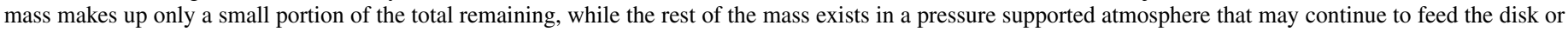

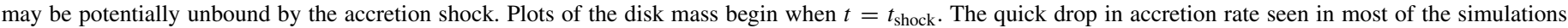
occurs around the time of shock formation.

(A color version of this figure is available in the online journal.) 

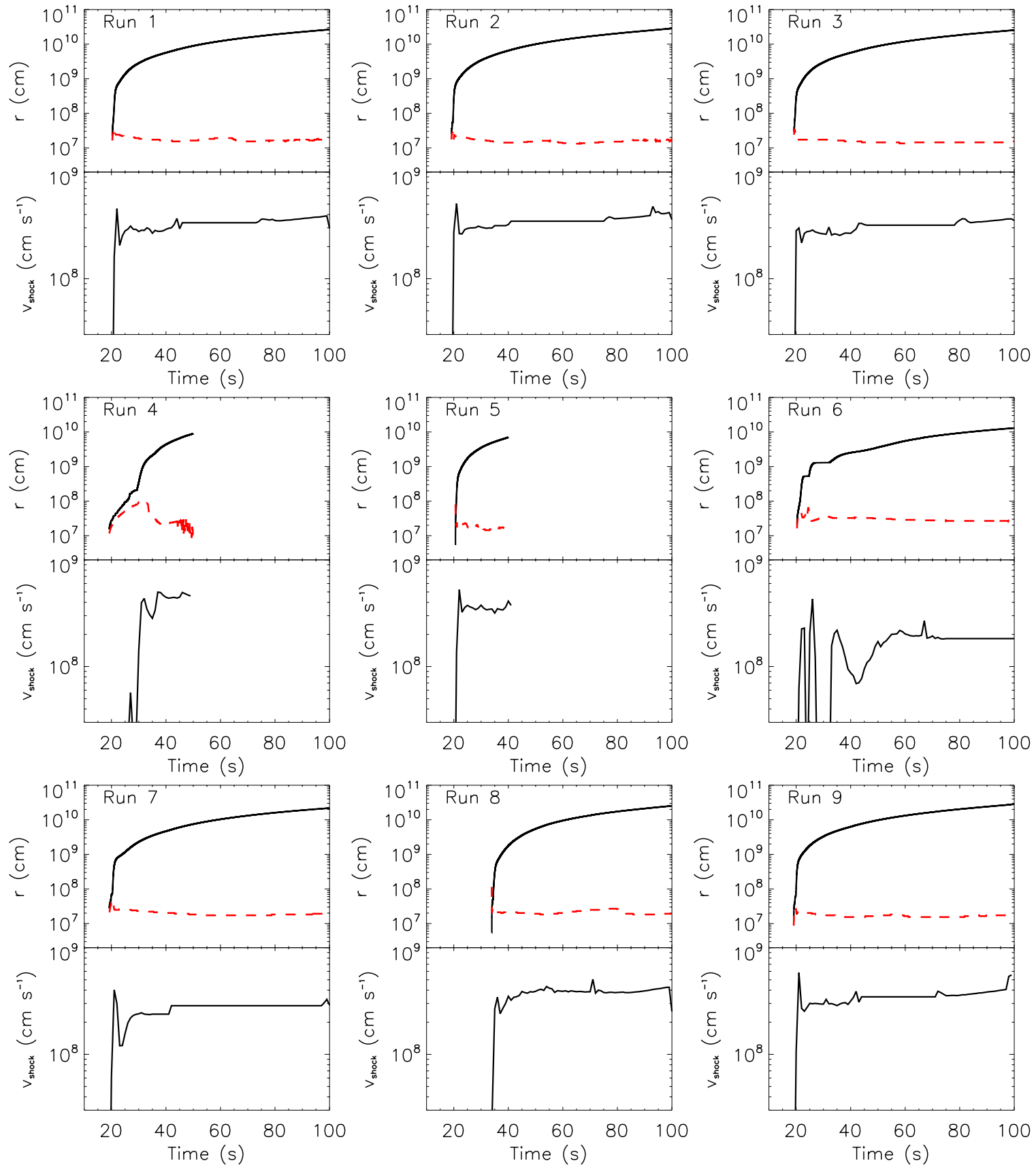

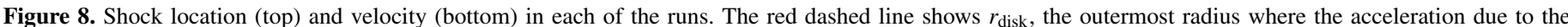

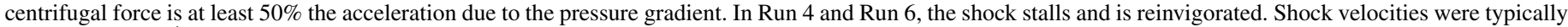
$2000-4000 \mathrm{~km} \mathrm{~s}^{-1}$. The small fluctuations in the shock velocity are numerical artifacts of the discreteness in our shock detection algorithm.

(A color version of this figure is available in the online journal.)

simulation declines monotonically. In most runs (1-4 and 7-9) the disk mass declines to $M_{\text {disk }} \lesssim 10^{-5} M_{\odot}$ by the end of the simulation, while in Run 6, the mass at the end of the simulation is somewhat larger but still very small, $M_{\text {disk }} \sim 3 \times 10^{-4} M_{\odot}$.

Specific angular momentum as a function of radius is shown in Figure 9. In the initial angular momentum profile of the model, compositional boundaries coincide with discontinuities in the profile, but in 16TI these occur only at mass coordinates that are accreted directly onto the black hole, prior to the initial circularization. The angular momentum profile of the mass shells remaining at initial circularization is monotonically increasing and most of the remaining mass has nearly the same angular momentum, $\sim(1-2) \times 10^{17} \mathrm{~cm}^{2} \mathrm{~s}^{-1}$. This implies that the shocked atmosphere has nearly uniform specific angular momentum everywhere except at the radii where the timescale on which the viscous torque transport angular momentum is shorter than the time since circularization. At $(25-50)$ s, there is a mild, sub-Keplerian inward downturn in $\ell(r)$ at $r \lesssim 1000 \mathrm{~km}$. Angular momentum transport is too slow within the initial $\sim 100 \mathrm{~s}$ to affect the radii $\gtrsim 10^{4} \mathrm{~km}$.

\footnotetext{
4 Stellar models exist in which nonmonotonicity is pronounced. This can produce an interesting variability of the central accretion rate (e.g., López-Cámara et al. 2010; Perna \& MacFadyen 2010).
} 


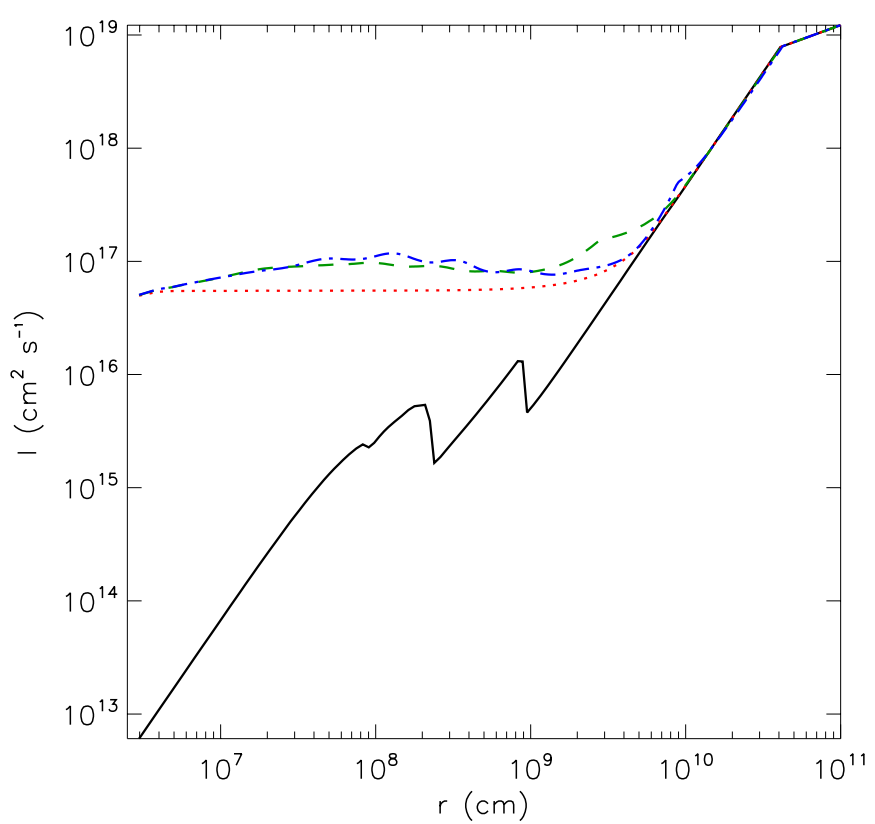

Figure 9. Specific angular momentum, $\ell$, as a function of radius in Run 1 at $t=0 \mathrm{~s}$ (black, solid), $t=15 \mathrm{~s}$ (red, dotted), $t=25 \mathrm{~s}$ (green, dashed), and $t=50 \mathrm{~s}$ (blue, dot-dashed). The initial rotational profile for the stellar model shows large spikes at compositional boundaries. Early in the simulation, low angular momentum material is quickly accreted.

(A color version of this figure is available in the online journal.)

The mass accretion rate as a function of radius in Run 1 at $t=50 \mathrm{~s}$ is shown in Figure 2. The accretion rate is independent of radius for $r \lesssim 2000 \mathrm{~km}$, which is the radii where the angular momentum profile has relaxed to a viscous quasi-equilibrium. The analytic expectation, given in Equation (52), is shown as well. Figure 10 shows the radial velocity $v_{r}$, angular velocity $v_{\phi}$, and Keplerian velocity as a function of radius at $t=18 \mathrm{~s}$, just

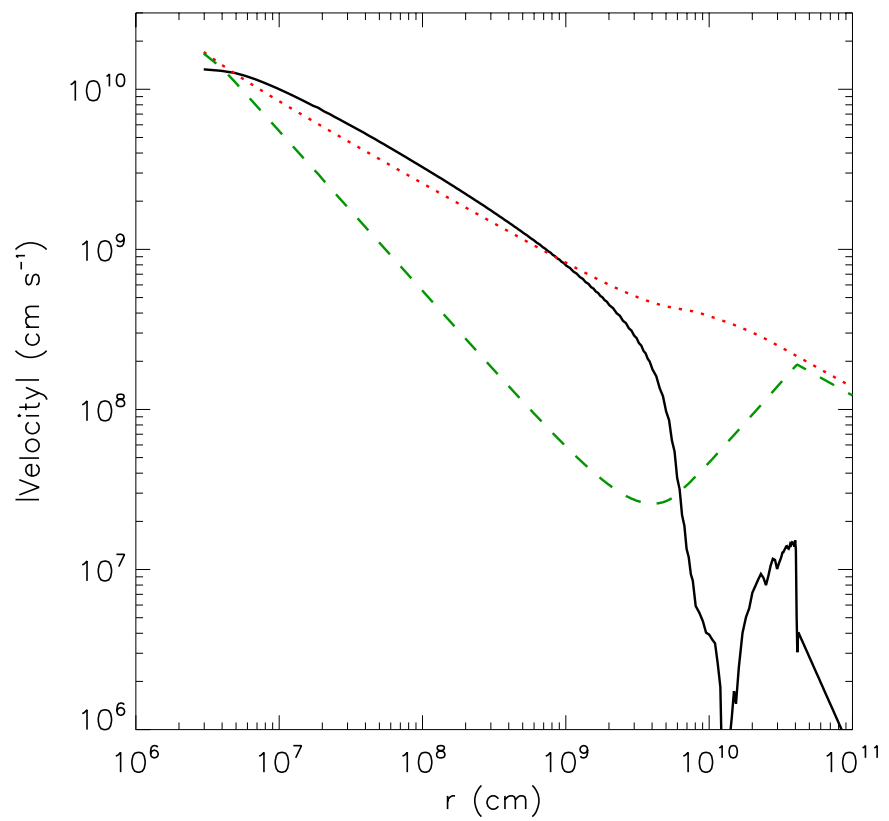

as material begins to circularize outside of the black hole, and at $t=30 \mathrm{~s}$, after an accretion disk has formed. At $t=18 \mathrm{~s}$, the velocity $v_{\phi}$ reaches the Keplerian value at the innermost radii.

Throughout the simulations, we tracked the value of our estimate of the vertical ( $z$-directed) pressure scale height-toradius ratio $\tilde{H}_{z} / r$, as described in Section 2.6. When the estimated ratio is below one-half, this indicates that in two dimensions, the flow should be disk-like, and when $\tilde{H}_{z} / r \ll 0.5$, the flow is a geometrically thin disk. We found that $\tilde{H}_{z} / r$ is below 0.5 but is still always above a minimum of 0.3 everywhere, except in Run 4, which had the lowest viscosity. The disk-like radii where the vertical pressure scale height-to-radius ratio is below one-half are $r \lesssim 200 \mathrm{~km}$ immediately following circularization and shrink to $r \lesssim 100 \mathrm{~km}$ by the end of the simulation. In Run 4 with a reduced viscous stress-to-pressure ratio $\alpha$, neutrino cooling drove the disk to be geometrically thin, where $H_{z} / r \lesssim 0.3$ in the inner $r \lesssim 500 \mathrm{~km}$. In the innermost zone in Run $4, H_{z} / r=0.1$ at $t=20 \mathrm{~s}$, the lowest seen in any simulation. By $t=35 \mathrm{~s}$, no thin disk is present. In Figure 11 we show the value of $\Xi_{\text {mod }}$ defined in Equation (44) throughout the simulation in Runs 1, 4, and 6; it does not drop below $\sim 0.77$. Only in Run 4 is a genuinely thin accretion disk present, and there it is limited to small radii. The outer radius of the thin disk decreases as the neutrino luminosity drops (see Section 3.3). We attribute the observed moderate thinning of the accretion flow to the cooling of the flow by the photodisintegration of helium nuclei into free nucleons, and in Run 4, the additional contribution of neutrino cooling is also significant.

\subsection{Energy Transport}

To understand the energetics of the accretion flow in a collapsar, we need to consider the transport of mechanical, thermal, and nuclear binding energy, as well as the loss to neutrino emission. Before turning to energy transport, we

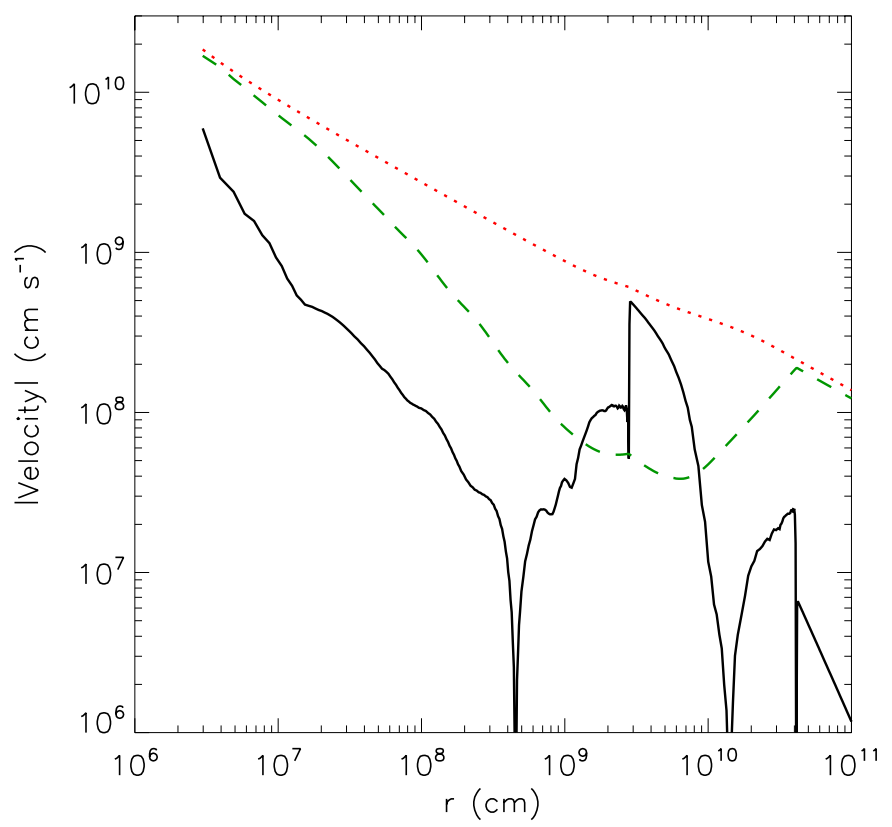

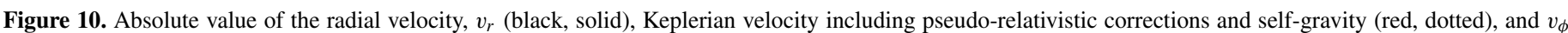

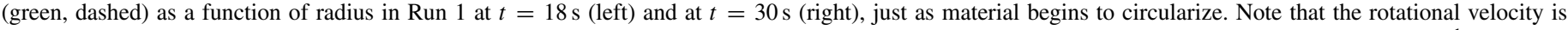

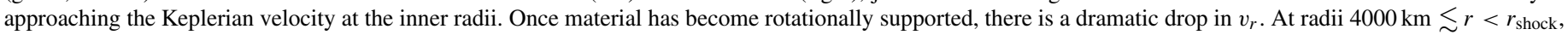
the radial velocity is positive, indicating an outflow.

(A color version of this figure is available in the online journal.) 

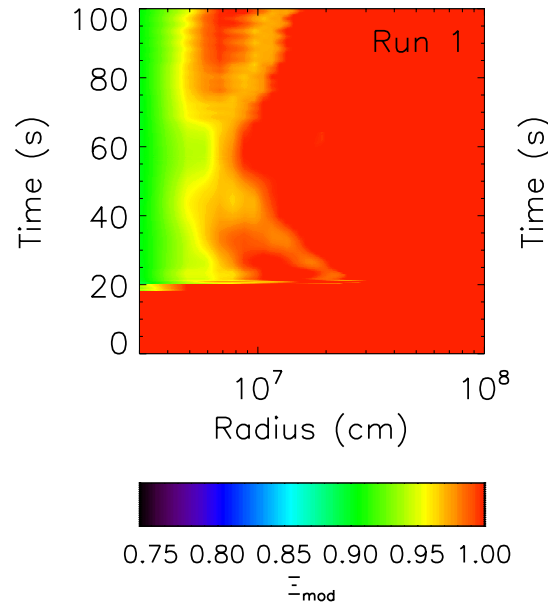
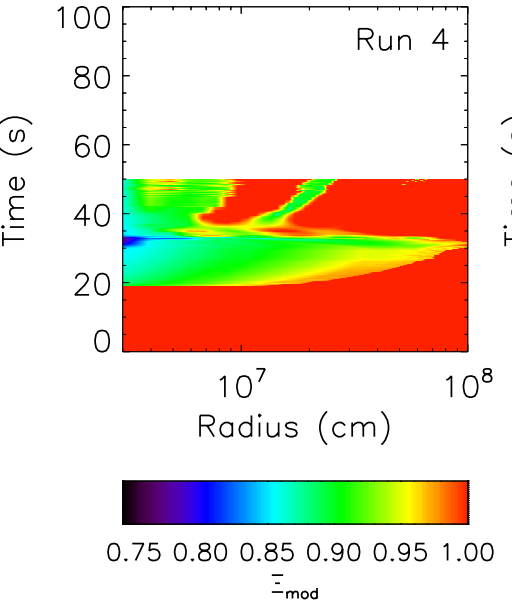
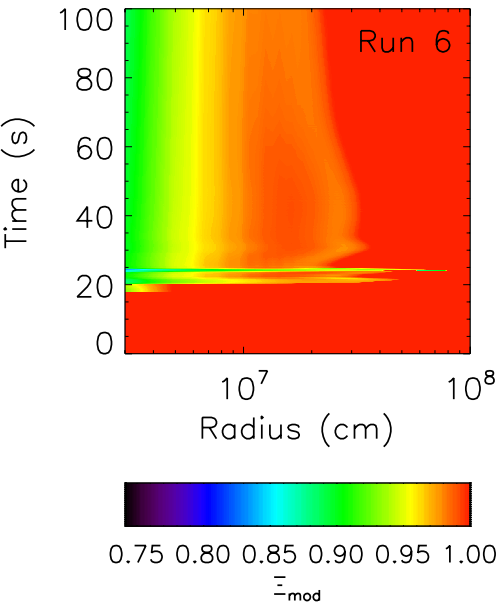

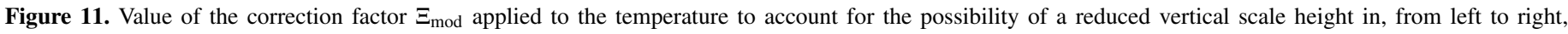

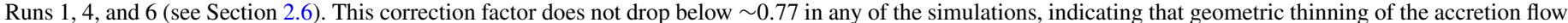
is a relatively weak effect. The correction is only applied in regions where $\mathbf{a}_{\text {cent }}>0.5 \mathbf{a}_{\text {pres }}$, which occurs only for $r<1000 \mathrm{~km}$; thus $\Xi_{\text {mod }}=1.0$ for $r>1000 \mathrm{~km}$. (A color version of this figure is available in the online journal.)

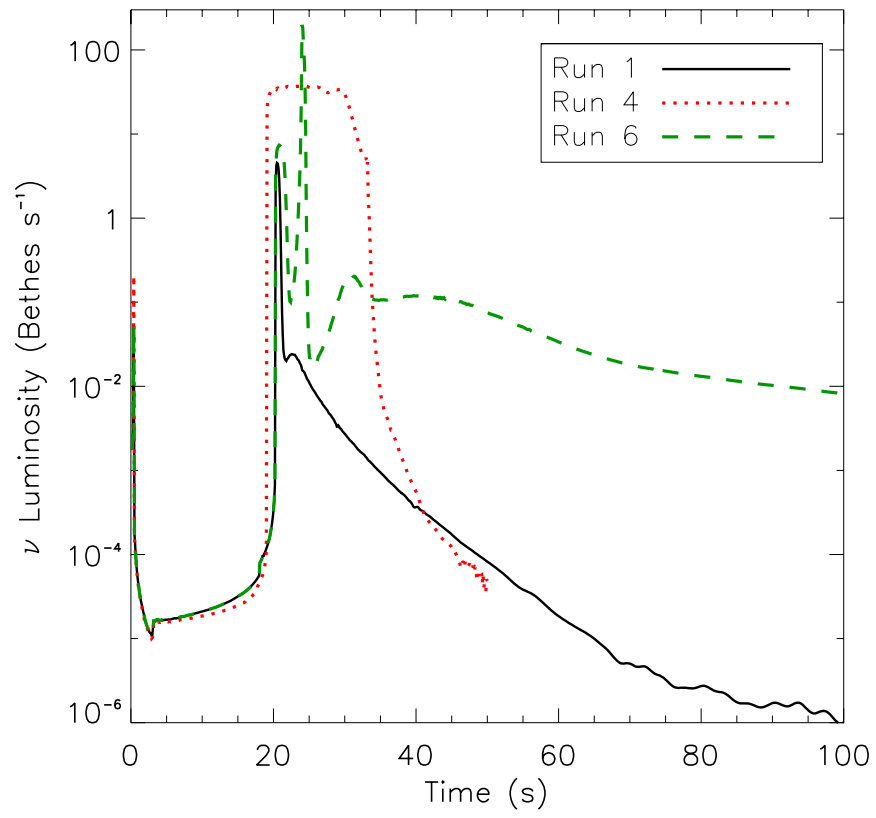

Figure 12. Neutrino luminosity integrated over the entire computational domain in representative Runs 1, 4, and 6 (see Section 2.4). The peak in luminosity occurs shortly after the formation of the accretion shock. Note that we do not capture neutrino emission from the region $r<25 \mathrm{~km}$, where much neutronization and peak neutrino luminosity is expected to occur in the first few seconds after the formation and collapse of the iron core. The total neutrino luminosities integrated over the entire simulation in Runs 1, 4, and 6 were 2.7, 419.3 , and $83.9 \times 10^{51} \mathrm{erg}$, respectively.

(A color version of this figure is available in the online journal.)

discuss the neutrino losses, which turn out to be not significant in the regime we consider.

The integrated neutrino luminosity is dominated by the emission from the inner $\sim 100 \mathrm{~km}$. The luminosity as a function of time in the representative Runs 1, 4, and 6 is shown in Figure 12. In simulations with $\alpha=0.1$, neutrino luminosities integrated over the entire computational domain peaked immediately following shock formation at $\sim(1-200) \times 10^{51} \mathrm{erg} \mathrm{s}^{-1}$. The peak luminosity lasted anywhere from less than a second in the runs with high peak luminosities to a few seconds in the runs with low peak luminosities. After the peak, the luminosity decays first very rapidly until it has dropped to $\sim 10^{50} \mathrm{erg} \mathrm{s}^{-1}$, and then continues to decay approximately exponentially by several orders of magnitude to settle at $\sim\left(10^{-6}\right.$ to $\left.10^{-5}\right) \mathrm{erg} \mathrm{s}^{-1}$ after $\sim 50 \mathrm{~s}$. The sharp luminosity peak is an artifact of the abrupt nature of shock formation in our 1.5D dimensional treatment and is probably not physically significant. The total energy that would be deposited by an absorption of the emitted neutrinos, which we do not calculate, is negligible.

Now turning to energy transport, we examine the radial transport of all forms of energy, the thermal and kinetic energies, the nuclear binding energy, and the gravitational potential energy. The gravitational potential energy is a nonlocal functional of the mass distribution. However, ignoring relativistic effects, one can define the gravitational potential energy per unit volume to be $\rho\left(\Phi_{\mathrm{BH}}+(1 / 2) \Phi_{\mathrm{s}}\right)$, where $\Phi_{\mathrm{BH}}$ is the gravitational potential of the black hole which we define via $\Phi_{\mathrm{BH}}(r) \equiv \int_{r}^{\infty} g_{\mathrm{BH}}\left(r^{\prime}\right) d r^{\prime}$ with $g_{\mathrm{BH}}$ given in Equation (9) and $\Phi_{\text {self }}$ is that of the selfgravity of the star. Then, $\rho v_{r} \Phi$, where $\Phi=\Phi_{\mathrm{BH}}+\Phi_{\text {self }}$, can be interpreted as the flux of gravitational energy advected by the fluid, but one must additionally include the flux of gravitational energy transported by self-gravity (see, e.g., Binney \& Tremaine 2008, their Appendix F), which equals

$$
F_{\text {grav,self }}=\frac{1}{8 \pi G}\left(\Phi_{\text {self }} \nabla \frac{\partial \Phi_{\text {self }}}{\partial t}-\frac{\partial \Phi_{\text {self }}}{\partial t} \nabla \Phi_{\text {self }}\right) .
$$

This term is significant only in the outer envelope of the star. The rate with which the sum total of these energies is transported radially is given by

$$
\begin{aligned}
\dot{E}= & 4 \pi r^{2}\left[\rho v_{r}\left(\epsilon+\epsilon_{\text {nuc }}+\frac{P}{\rho}+\frac{1}{2} v_{r}^{2}+\frac{1}{2} \frac{\ell^{2}}{r^{2}}+\Phi\right)+F_{\text {grav, self }}\right. \\
& \left.-\rho \nu \ell \frac{\partial \Omega}{\partial r}+F_{\text {conv }}+F_{\text {nuc, } \text { mix }}\right],
\end{aligned}
$$

where the convention is such that $\dot{E}>0$ implies the transport of positive energy outward, opposite from the convection employed in the definition of the mass accretion rate $\dot{M}$. Here, $-\rho \nu \ell \partial \Omega / \partial r$ is flux of energy transported by the viscous stress.

Specific entropy as a function of radius at several times in Runs 1 and 2 is shown in Figure 5. After the formation of the accretion shock and the rotationally supported flow, 


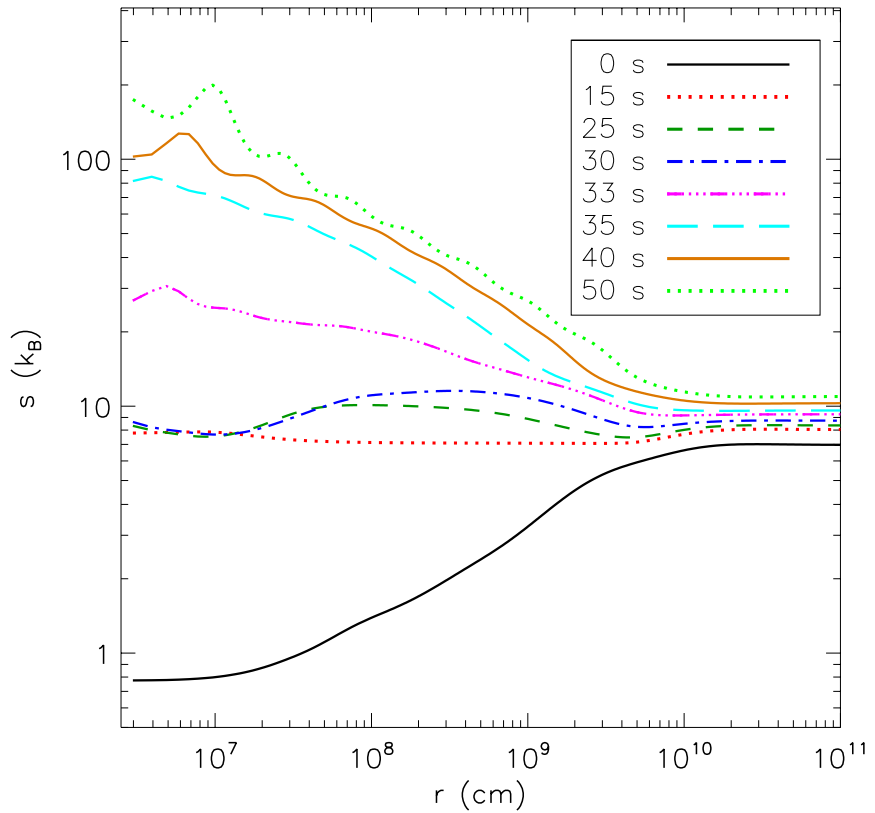

Figure 13. Smoothed entropy $s_{\text {smooth }}$ per baryon in units of the Boltzmann constant $\left(k_{\mathrm{B}}\right)$ at various times in the low-viscosity Run 4 (cf. Figure 5). Unlike in the other runs, here a negative specific entropy gradient is not seen immediately after the fluid comes into radial force balance. Even by $t=30 \mathrm{~s}$ the fluid is still stable against convection; neutrino cooling prevents the early rise of convective instability. Once the neutrino luminosity begins to drop around $t=33 \mathrm{~s}$, entropy in the post-shock region begins to rise, bringing about strong entropy inversion. By the end of the simulation, Run 4 has the largest value of entropy seen in any of the simulations.

(A color version of this figure is available in the online journal.)

viscous dissipation heats the fluid, thus producing a negative entropy gradient in the shock downstream. The negative specific entropy gradient extends almost to the shock front, and thus the energy injected at small radii can travel to raise the entropy of the entire post-shock region. Figure 13 shows the specific entropy in Run 4. Here, the high neutrino luminosity after the accretion shock has formed keeps the entropy in the post shock region relatively low. For the first $\sim 10 \mathrm{~s}$ after shock formation, no specific entropy inversion is seen, and the fluid is stable against convection. When the neutrino luminosity begins to drop around $t \approx 33 \mathrm{~s}$, the entropy rises, a negative specific entropy gradient appears in the post-shock region, and convection starts transporting the viscously dissipated energy outward.

Figure 14 plots the net transport rate $\dot{E}$ and the various constituent terms in Run 2 at $t=30 \mathrm{~s}$; the radii and other observables quoted in the remainder of this section will be specific to this particular simulation snapshot and will vary across different simulations and different times within a simulation. Approximate radial independence of the energy transport rate, $\partial E / \partial r \approx 0$ for $200 \mathrm{~km} \lesssim r \lesssim 4000 \mathrm{~km}$, where the transport rate is positive $\dot{E} \approx 10^{50} \mathrm{erg} \mathrm{s}^{-1}>0$, is indicative of quasisteady-state accretion. At larger radii, $r \gtrsim 5000 \mathrm{~km}$, where the inner inflow gives way to an outer outflow-a precursor of the brewing explosion-no quasi-steady state is present and the fluid variables evolve on the dynamical time in the wake of the expanding shock. At small radii, $r \lesssim 100 \mathrm{~km}$, where one expects a steady state, the curve $\dot{E}(r)$ exhibits a small positive gradient, as well as a sawtooth consistent with that seen in the accretion rate $\dot{M}(r)$. The constancy of the plotted energy transport rate is contingent on an accurate cancellation of the other transport terms. We suspect that the observed nonconstancy is

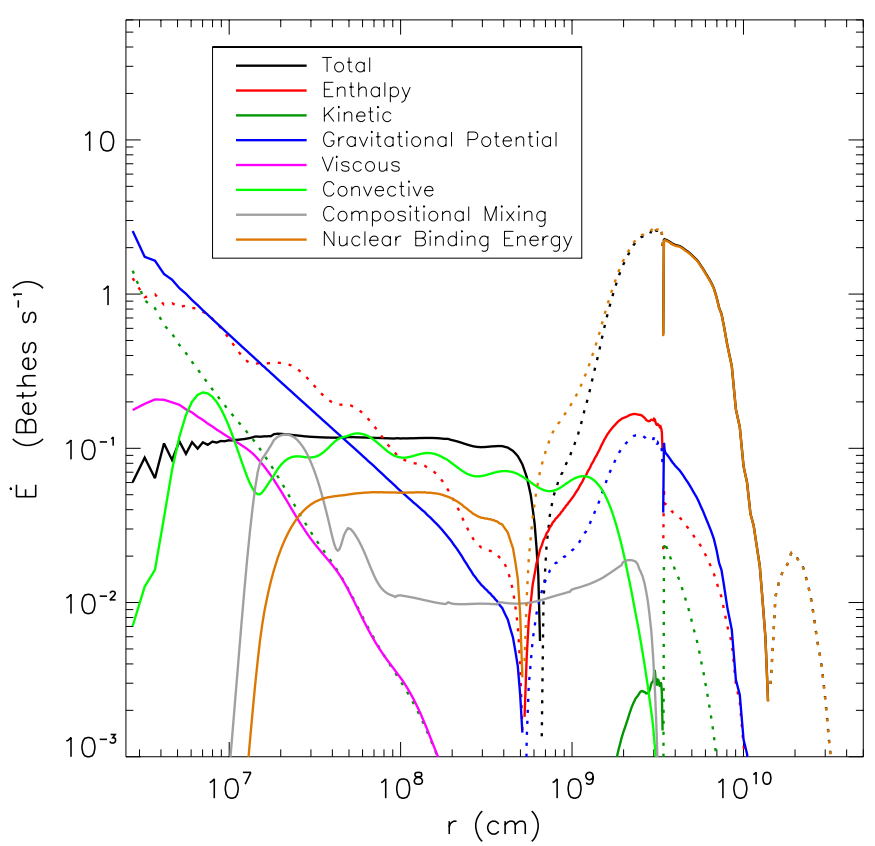

Figure 14. Total and partial energy transport rates in Run 2 at $t=30 \mathrm{~s}$ (see Section 3.3 and Equation (55)). The curves show $\dot{E}$ (black), the enthalpy advection rate $4 \pi r^{2} v_{r}(\rho \epsilon+P)$ (red), the kinetic energy advection rate $2 \pi r^{2} v_{r} \rho\left(v_{r}^{2}+\ell^{2} / r^{2}\right)$ (green), the gravitational potential energy transport rate $4 \pi r^{2}\left(\rho v_{r} \Phi+F_{\text {grav, self }}\right)$ (dark blue), the rate of energy transport by the viscous stress $-4 \pi r^{2} \rho \nu \ell \partial \Omega / \partial r$ (pink), the rate of thermal energy transport by convection $4 \pi r^{2} F_{\text {conv }}$ (green), the nuclear energy transport rate associated with convective compositional mixing $4 \pi r^{2} F_{\text {nuc, mix }}$ (gray), and the nuclear binding energy advection rate $4 \pi r^{2} v_{r} \rho \epsilon_{\text {nuc }}$ (orange). Negative values are indicated by dotted lines.

(A color version of this figure is available in the online journal.)

arising from relatively small inconsistencies in the discretization or gravitational source terms in FLASH and in the calculation of the gravitational energy during post-processing.

At $r \lesssim 1000 \mathrm{~km}$, the inward advection of thermal and kinetic energy dominates over the outward transport by convection and the viscous stress. Therefore, the innermost flow is an advection-dominated accretion flow (ADAF; Narayan \& Yi 1994, 1995; Blandford \& Begelman 1999). At $r \gtrsim 1000 \mathrm{~km}$, the outward transport of thermal energy by convection dominates the inward transport by advection and this region is thus a convection-dominated accretion flow (CDAF, see, e.g., Stone et al. 1999; Igumenshchev et al. 2000; Blandford \& Begelman 2004). Inward nuclear binding energy advection and nuclear compositional mixing both act to transport the total energy outward if one counts the negative nuclear binding energy in the total energy budget. Convection transports energy from the ADAF-CDAF transition radius where the magnitude of the enthalpy advection flux $\sim\left|v_{r}(\rho \epsilon+P)\right|$ equals the convection flux $F_{\text {conv }}$ to the shock radius $r_{\text {shock}}$. In Figure 14 the former is at $r_{\mathrm{ADAF}} \approx 1000 \mathrm{~km}$ and the latter is at $r_{\text {shock }} \approx 3.5 \times 10^{4} \mathrm{~km}$. In Run 1 and similar runs, $r_{\mathrm{ADAF}}$ increases very slowly from $\sim 1000 \mathrm{~km}$ to $\sim 2000 \mathrm{~km}$ from shock formation until $t=100 \mathrm{~s}$. In Run 4 , the radius is $\sim 200 \mathrm{~km}$ throughout the simulation, and in Run 6 , the radius grows from $\sim 5000 \mathrm{~km}$ to over $\sim 10^{4} \mathrm{~km}$ in the course of the simulation.

We suspect that the location of $r_{\mathrm{ADAF}}$ determines the amount of energy that can be carried to the shock front, and that in turn, the ADAF-CDAF transition radius is primarily a function of the convective efficiency $\xi_{\text {conv }}$ and the viscous stress-topressure ratio $\alpha$. Simulations with larger values of $\xi_{\text {conv }}$ resulted 


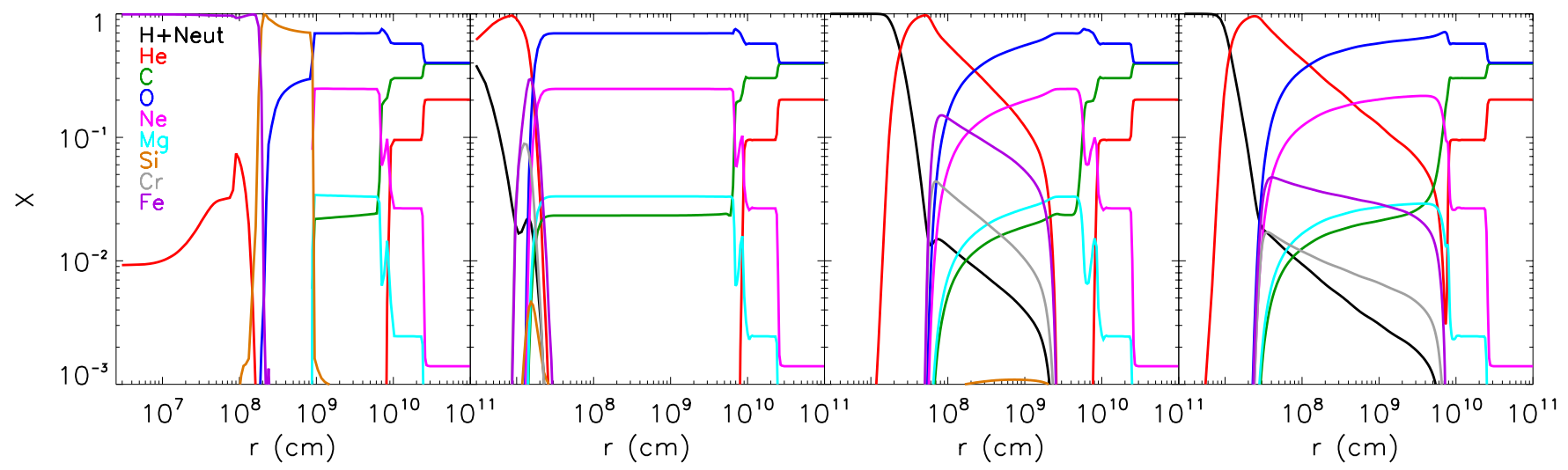

Figure 15. Abundances of the common elements summed over all isotopic species at (left to right) $t=0,15,25,50 \mathrm{~s}$ in Run 1 .

(A color version of this figure is available in the online journal.)

in stronger shocks and larger amounts of unbound material. The convective compositional mixing parameter $\xi_{\text {conv,mix }}$ had little effect on the final outcome of our simulations. Turning to the viscosity parameter, Run 3 with a large values of $\alpha$ produced a somewhat less energetic shock with less unbound material at the end of the simulation. Run 4, the simulation with the lowest viscosity, produced the most energetic explosion, even in the presence of more pervasive cooling by neutrino emission and by photodisintegration in the low- $\alpha$ regime; these flows are denser and hotter (see, e.g., Popham et al. 1999; Chen \& Beloborodov 2007). In Milosavljević et al. (2012), we show that $r_{\mathrm{ADAF}}$ is expected to be smaller under low viscosity conditions because the advective luminosity is proportional to $v_{r}$, which is proportional to $\alpha$. This trend is reproduced in the present simulations.

\subsection{Nuclear Composition of the Flow}

Our simplified treatment of nuclear compositional transformation, which entails relaxation to NSE on a temperaturedependent timescale, is designed to track the impact of nuclear photodisintegration and recombination on the thermodynamics of the flow. However, it does not allow the computation of the ultimate nucleosynthetic output in the presence of out-of-NSE burning. Thus, the results presented here can only be understood in light of the limitations of the method. ${ }^{5}$ It is also worth recalling that we do not calculate neutronization that could modify the proton-to-nucleon ratio $Y_{e}$.

In the hottest, innermost accretion flow, photodisintegration of heavy nuclei saps energy that could otherwise be transported by convection to larger radii to energize the shocked envelope. However, once the nuclei are broken down, convective mixing can dredge up free nucleons to larger and cooler radii, where they can recombine and heat the fluid locally. Figure 6 shows the mean atomic mass $\bar{A}$ as a function of radius at various times in Run 1 and Run 2. The mean atomic mass drops below 4 in the innermost (200-300) km. The positive gradient in $\bar{A}$ seen in portions of the convective region would in the Ledoux picture enhance the convective energy flux, but our Schwarzchild treatment of convection does not capture this effect. We argue in Section 2.9 that since the nuclear

\footnotetext{
5 Metzger (2012) modeled accretion disks associated with the mergers of white dwarfs and neutron stars or black holes. He found that nuclear processes taking place at temperatures $T \lesssim 4 \times 10^{9} \mathrm{~K}$ may lead to significant heating in the resulting outflows. The relaxation to NSE we employ underestimates the heating due to out-of-NSE nuclear recombination.
}

timescale is shorter than the convective timescale at radii where Ledoux convection implies an enhanced energy transport, the nuclear compositional transformation inside convective cells, not considered in the Ledoux treatment, should dominate. Lacking a theory of convection in this regime we adhere to the simpler Schwarzschild parameterization.

In Figure 15, we show the mass-weighted abundances of the most common elements in our simulations in Run 1 at various times. By $t=30 \mathrm{~s}$, again, the inner $200 \mathrm{~km}$ is made up almost entirely of free nucleons in nearly equal portions, as $Y_{e} \approx 0.5$ everywhere. The effect of convective compositional transport of the reprocessed nuclear species - the free nucleons, helium, and iron-from the hot innermost accretion flow is seen in the power-law tails extending to near the location of the shock in the right panels.

Although in our calculations we do not allow the evolution of $Y_{e}$, we can still speculate about the effects of neutronization. Chen \& Beloborodov (2007) computed the structure of timeindependent accretion disks around Kerr black holes including the effects of pair capture and neutronization. In their models with $\alpha=0.1$, the same as our fiducial viscous stress-to-pressure ratio, at the radii where $\rho \sim 10^{7} \mathrm{~g} \mathrm{~cm}^{-3}$, corresponding to the density in the innermost disk in our simulations, they find $Y_{e} \approx 0.5$, the same as in our non-neutronizing treatment. In their models with $\alpha=0.01$, however, Chen \& Beloborodov (2007) find that at densities corresponding to the innermost disk in our simulations, significant neutronization was in effect and $Y_{e}$ dropped well below neutron-proton equality. It is therefore possible that in the very innermost regions of the disks in our Run 4 with a low viscosity $\alpha=0.025$, the true value of $Y_{e}$ should be lower than we assume. This would modify the abundances and thermodynamics of the portion of the flow in NSE. The key question of consequence for the viability of the mechanism we propose for the production of luminous supernovae is, will the neutron-rich material pollute larger radii and drive a tendency toward the synthesis of iron instead of ${ }^{56} \mathrm{Ni}$ ? Because this neutronization only seems to be most significant in the hottest innermost regions, where neutrino cooling is efficient and the flow is predominantly rotationally supported, it is possible that most of the neutron-enhanced material would be advected into the black hole. This is a quantitative question that can be answered only with multidimensional simulations.

In Figure 16, we show the location of $r_{\mathrm{NSE}}$, the largest radius at which nuclear compositional transformation, in the form of gradual convergence toward NSE, is taking place in our Run 1. This is the only region in which our calculations will capture 


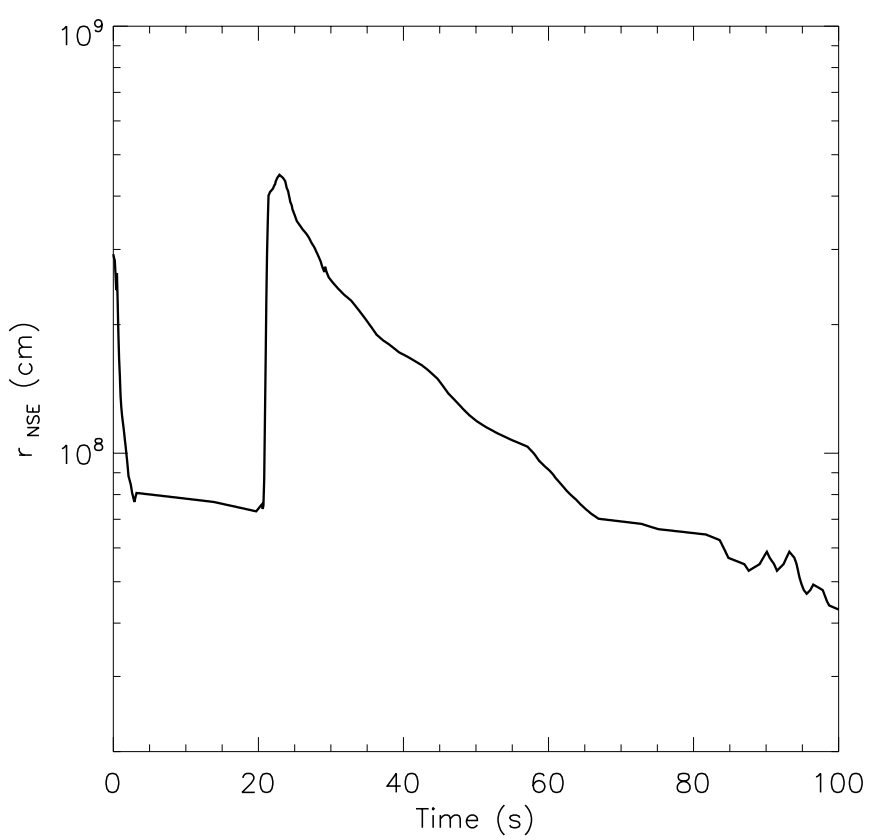

Figure 16. Location of $r_{\mathrm{NSE}}$, the largest radius at which nuclear compositional transformation, in the form of gradual convergence toward NSE, is taking place in our Run 1; this radius is closely approximated with the radius where the temperature crosses $\approx 3 \times 10^{9} \mathrm{~K}$.

nucleosynthesis and photodisintegration. Effectively, it is the radius at which $T>3 \times 10^{9} \mathrm{~K}$. Early in the simulation, the hot stellar core accretes into the black hole, and $r_{\mathrm{NSE}}$ quickly drops from $\approx 3000 \mathrm{~km}$ to $\approx 800 \mathrm{~km}$. After the shock forms, additional heating in the shock and by viscous dissipation rapidly increases $r_{\mathrm{NSE}}$ and it peaks at $\approx 4000 \mathrm{~km}$. As the density and temperature drop and the viscous heating rate decreases, the inner regions of the star begin to cool, and $r_{\text {NSE }}$ declines again.

In Figure 17, we show the total mass of various nuclear species in the entire simulation domain as a function of time. The most notable change is the dip in the mass of iron-group elements. The initial decrease in the iron-group mass is the accretion of the core of the star onto the black hole. After shock formation, a rapid increase in the amount of free nucleons is seen, in addition to production of additional helium and iron-group elements. In Table 2, we show the total amount of newly fused Fe-group elements present at the end of the simulation, which fall in the range of $0.02 M_{\odot}-0.09 M_{\odot}$. Since we do not calculate the outof-NSE burning in convectively dredged up material, at least a fraction of the extended helium tail seen in Figure 15 could be expected to burn into iron and thus the iron-group mass in Figure 17 and Table 2 can be interpreted as a lower limit.

\subsection{Prospects for Explosion}

The total thermal and mechanical energy, which we also refer to as the total binding energy, present on the grid was computed for each simulation via

$$
E_{\text {bind }}=\int_{r_{\min }}^{r_{\max }} \rho\left(\epsilon+\frac{1}{2} v_{r}^{2}+\frac{1}{2} \frac{\ell^{2}}{r^{2}}+\Phi_{\mathrm{BH}}+\frac{1}{2} \Phi_{\text {self }}\right) 4 \pi r^{2} d r
$$

and is shown in Figure 18. A positive binding energy indicates a potential for explosion. Runs 1, 2, 3, 4, 5, 8, and 9 acquired a positive total binding energy $E_{\text {bind }} \sim(3.5-6.2) \times 10^{50} \mathrm{erg}$ by the end of the simulation, indicating the potential for explosion. These runs have $\xi_{\text {conv }} \geqslant 2$ in common. Run 7

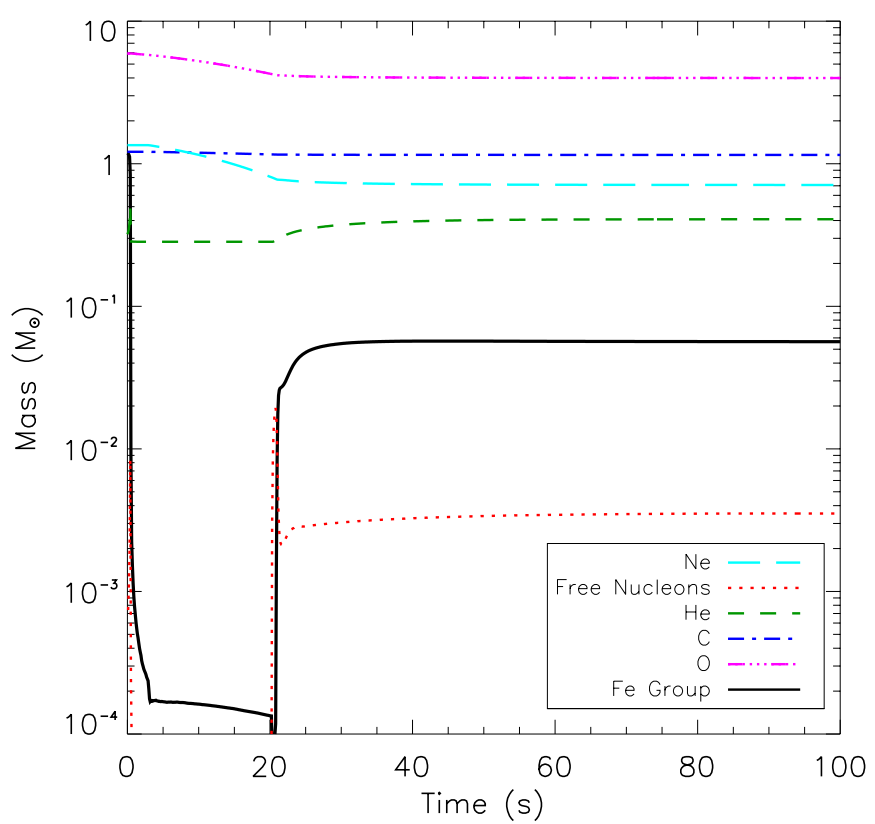

Figure 17. Mass of the most common elements summed over isotopic species in Run 1 as a function of time. Here, Fe represent all the isotopes of the iron group. At the start of the simulation, there is a large dip in the amount of iron group elements, due to the accretion of the iron core. After shock formation, $\sim 0.05 M_{\odot}$ of iron group elements are synthesized.

(A color version of this figure is available in the online journal.)

with $\xi_{\text {conv }}=1$ reached marginally unbound condition with $E_{\text {bind }} \sim(0.5-1) \times 10^{50} \mathrm{erg}$. Run 6 with $\xi_{\text {conv }}=0.5$ remained gravitationally bound throughout the entire simulation.

The total unbound mass in each simulation was calculated by summing the masses of any fluid element with a positive value of $E_{\text {bind }}$ and a positive radial velocity. The total unbound masses in each simulation defined by this diagnostic are shown in Table 2 . This criterion does not take into account any interaction that unbound and bound material might have subsequent to the measurement. This criterion also neglects future energy gain or loss from nuclear processes. The location and velocity of the outward moving accretion shock is shown in Figure 8. In exploding models, the typical shock velocities were $(2000-4000) \mathrm{km} \mathrm{s}^{-1}$. In Run 4 and Run 6, the shock stalled or slowed, and later restarted once or several times. In Figure 19, we show the evolution of various Lagrangian mass coordinates in Run 1 throughout the simulation. Once the shock moves beyond $\sim 4000 \mathrm{~km}$, the infalling material obtains a positive velocity once it reaches $r_{\text {shock }}$.

These results suggest that a high convective efficiency is required for sufficient transfer of energy from the inner accretion flow to the envelope to unbind the envelope. Simulations with higher values of $\xi_{\text {conv }}$ had relatively larger shock velocities and amounts of unbound mass at the ends of their simulations, and Run 4 with a lower $\alpha$ had the largest value of $E_{\text {bind }}$. Run 8 with reduced initial specific angular momentum produced an explosion comparable to that seen in the fiducial model.

\section{OBSERVATIONAL SIGNATURES AND PROGENITOR TYPES}

Modeling of light curves and spectra of supernovae associated with LGRBs has yielded information about the nature of these explosions. The mass of ${ }^{56} \mathrm{Ni}$ present in the supernova ejecta is easily estimated from the light curve by fitting simple radioactive decay models. The velocity of the ejecta is inferred 


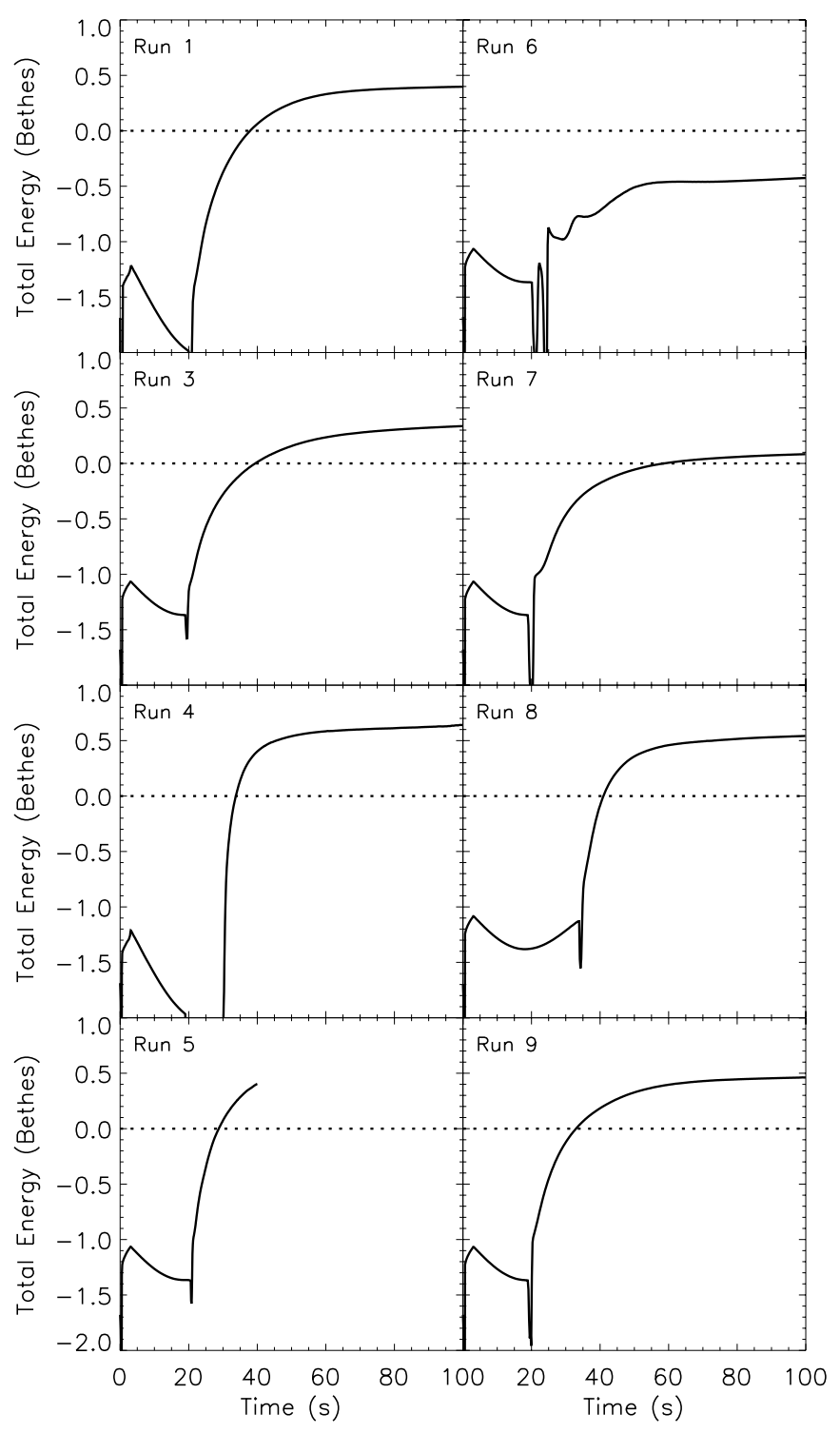

Figure 18. Total binding energy on the simulation grid in Runs 1 and 2-9. Simulations in which the convective efficiency factor, $\xi_{\text {conv }} \geqslant 0.5$, reached a positive total binding energy by the end of the simulation. The large, brief dips in energy visible in some simulations around the time of shock formation are primarily due to cooling by neutrino emission. The large dip seen in Run 4 is also predominantly due to neutrino cooling and has a minimum of $E_{\text {bind }}=-1.5 \times 10^{52} \mathrm{erg}$.

from observed line widths. Then, in a standard approach, the kinetic energy and mass of the ejecta are derived by comparing the inferred ${ }^{56} \mathrm{Ni}$ yield with that implied by one-dimensional hydrodynamic models in which a spherical shock wave is introduced by hand (by the action of a hypothetical "piston") into the progenitor's envelope. ${ }^{6}$ This approach relies on the hypothesis that ${ }^{56} \mathrm{Ni}$ is produced at the shock front, for which the shock must be very strong.

Our simplified simulations suggest an alternative scenario in which nucleosynthesis takes place in the accretion flow in the

\footnotetext{
6 In "piston" and "thermal bomb" models, an explosion is mimicked by injecting a large kinetic or thermal energy into a narrow shell over a relatively short $\lesssim 1$ s time interval (e.g., Jones et al. 1981; Woosley \& Weaver 1986; Arnett 1987; Shigeyama et al. 1987; Woosley et al. 1988; Thielemann et al. 1990; Woosley \& Weaver 1995; Limongi \& Chieffi 2003; Chieffi \& Limongi 2004; Young \& Fryer 2007; Fryer et al. 2008; Kasen \& Woosley 2009; Maeda \& Tominaga 2009; Joggerst et al. 2010; Dessart et al. 2011).
}

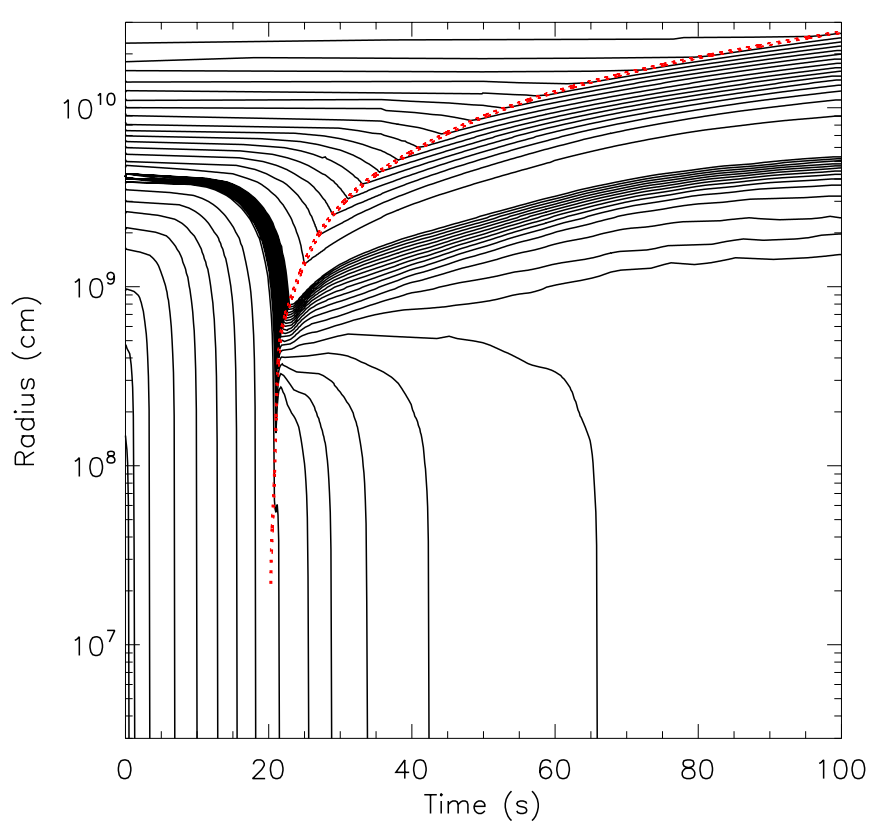

Figure 19. Evolution of the Lagrangian mass coordinates in Run 1 (black, solid). The location of $r_{\text {shock }}$ is also shown (red, dashed). The bifurcation between an inner inflow and an outer outflow occurs at $r \sim 4000 \mathrm{~km}$. This corresponds to a mass coordinate of $\sim 5.6 M_{\odot}$.

(A color version of this figure is available in the online journal.)

interior of the star, similar to the wind-nucleosynthesis models (e.g., Beloborodov 2003; Pruet et al. 2003, 2004; Nagataki et al. 2006; Surman et al. 2006; Maeda \& Tominaga 2009; Metzger 2012). Our results, however, suggest that the accretion flow is long lived, lasting tens or hundreds of seconds or longer, and so the nucleosynthesis can be sustained at lower densities than in the wind models, and its products can be delivered to the envelope by vigorous convection.

We also find that a supernova-like shock wave may be powered by the sustained input of accretion energy, without energization by neutrino energy deposition. The dynamics of the accretion-energy-powered shock wave is fundamentally different from that powered by a piston. It remains to be explored whether the accretion scenario will call for a modification of the standard approach to modeling the light curves and spectra of the supernovae that could be yielding black holes. We are thus somewhat reluctant to compare our results directly with observational inferences obtained with existing supernova models. Previous work has attributed kinetic energies of $\sim(2-50) \times 10^{51} \mathrm{erg}$ to supernovae associated with LGRBs (see, e.g., Woosley \& Bloom 2006; Hjorth \& Bloom 2011, and references therein). Our models come short of these energies, but they are consistent with the low energy end among the more typical Type Ib and Ic supernovae (see, e.g., Table 4 in Hamuy 2003). Unfortunately the simplified treatment of nuclear compositional transformation does not allow us to predict the ${ }^{56} \mathrm{Ni}$ synthesized in our models. We can only say that supernovae powered by collapsar accretion should exhibit a high degree of mixing of hydrostatic and explosive elements.

The shock velocities at $t=100 \mathrm{~s}$, when the shock is still in the interior of the envelope, in the models that achieve explosion, are $v_{\text {shock }} \approx 4000 \mathrm{~km} \mathrm{~s}^{-1}$. This is a half or smaller fraction of the commonly cited values for shock velocities measured in the observed supernovae. Of course, leading to the breakout of the stellar surface, the shock accelerates as it traverses the negative density gradient. The mass-weighted rms free 
expansion velocity inferred from the total energy at the end of the simulation is $v_{\mathrm{FE}} \sim\left(2 E_{\text {bind }} / M_{\text {unbound }}\right)^{1 / 2} \sim 3000\left(E_{\text {bind }} / 0.5 \times\right.$ $\left.10^{51} \mathrm{erg}\right)^{1 / 2}\left(M_{\text {unbound }} / 5 M_{\odot}\right)^{-1 / 2} \mathrm{~km} \mathrm{~s}^{-1}$, again lower than usually quoted for the observed supernovae.

Our initial model of choice was the $M_{\text {star }} \approx 14 M_{\odot}$ W-R model star 16TI of Woosley \& Heger (2006), evolved to pre-core-collapse from a $16 M_{\odot}$ main-sequence progenitor. The model 16TI is commonly used in LGRB investigations (e.g., Morsony et al. 2007, 2010; López-Cámara et al. 2009, 2010; Lazzati et al. 2010, 2011a, 2011b; Lindner et al. 2010; Nagakura et al. 2011a, 2011b), but it has been suggested that the progenitors of supernovae with confirmed association with LGRBs must be associated with the core collapse of more massive stars, perhaps with masses in the range of $(25-60) M_{\odot}$ or higher (e.g., Podsiadlowski et al. 2004; Smartt 2009, and references therein). However, predictions regarding the nature of the final remnant in such explosions are sensitive to the highly debated details of the explosion mechanisms in core collapse supernovae. Observational studies of the spatial distribution of Type Ic supernovae and GRBs in galaxies suggest that the respective progenitors should be at least $\sim 25 M_{\odot}$ and $\sim 43 M_{\odot}$ (Raskin et al. 2008; see also Larsson et al. 2007). It is of interest to note that simulations of neutron-star-powered explosions have been successful only in the lowest mass progenitors. The accretion powered mechanism we propose will operate in more massive progenitors that produce black holes. It is reasonable to speculate that in progenitors more massive than in our model, or with different internal structure, the explosion energies would be much higher than we find, more in line with the high energies of the LGRB supernovae. The long-term accretion in massive collapsar progenitors deserves further study.

\section{CONCLUSIONS}

We have conducted a series of hydrodynamic simulations of the viscous post-core-collapse accretion of a rapidly rotating $\sim 14 M_{\odot}$ W-R star of Woosley \& Heger (2006) onto the central black hole that we assumed was in place at the beginning of the simulation. The spherically symmetric simulations with rotation were carried out for $100 \mathrm{~s}$ and resolved the radii down to $25 \mathrm{~km}$, including the range of radii where the collapsing stellar material circularizes around the black hole. The simulations tracked cooling by neutrino emission and the relaxation to NSE in the hot inner accretion flow. The simulations also tracked convection and convective compositional mixing in the mixing length theory approximation. Finally, the simulations tracked viscous angular momentum transport and the associated heating in the flow. To explore the sensitivity to model parameters, we varied the initial angular momentum profile, convective energy transport and compositional mixing efficiencies, and the viscous stress-to-pressure ratio $\alpha$. Our main findings are as follows.

Lacking sufficient angular momentum to be rotationally supported around the black hole, the inner mass coordinates of the stellar envelope accrete unshocked onto the black hole. At $t \sim 20 \mathrm{~s}$, or later with reduced initial angular momentum, the first mass shell able to circularize around the black hole arrives. Once material becomes circularized, an accretion shock forms as the mass shells in near free fall interact with the rotationally supported material.

This shock front travels outward, leaving behind a mostly pressure supported, shock heated, convective stellar envelope. Only a very small fraction of the mass is predominantly rotationally supported; the rotationally supported, geometrically thick disk connects smoothly to the pressure supported, shock- heated envelope. The structure and energetics of the flow are governed by accretion mechanics. The energy dissipated by the viscous stress at the innermost radii, the radii smaller than some critical $r_{\mathrm{ADAF}}$, is advected into the black hole. The innermost flow is thus an ADAF. At larger radii, convection transports the dissipated energy outward, into the stellar envelope and toward the expanding shock front, implying that the outer flow is a $\mathrm{CDAF}$. The delivery of energy from $\sim r_{\mathrm{ADAF}}$ to the envelope proceeds for tens of seconds, and the total energies delivered are sufficient to produce supernovae, albeit not as energetic as the ones inferred in association with LRGBs.

We found that the final energy deposited into the envelope strongly depended on the efficiency of convective energy transport and depended somewhat on the viscous stress-to-pressure ratio $\alpha$. These two parameters strongly influence the location of the ADAF/CDAF transition, as we have explored with crude analytical arguments in Milosavljević et al. (2012). The low- $\alpha$ model has a hotter disk with more pervasive cooling by neutrino emission and nuclear photodisintegration, sapping energy from the final explosion. However, the low- $\alpha$ disk also has an $\mathrm{ADAF} / \mathrm{CDAF}$ transition at a smaller radius, potentially allowing for a higher convective luminosity.

For sufficiently high convective efficiencies, the stellar envelope was capable of obtaining positive total thermal and mechanical energies $\sim 0.5 \times 10^{51} \mathrm{erg}$, shock velocities $\sim 4000 \mathrm{~km} \mathrm{~s}^{-1}$, and unbound masses $\sim 6 M_{\odot}$. We suggest that the accretion powered mechanism, which is distinct from and possibly mutually exclusive with the standard neutron-star-powered "delayedneutrino" mechanism, could explain low-luminosity Type Ib and Ic supernovae, but multidimensional study is needed to pin down the true efficiency of convective energy transport and to estimate the expected ${ }^{56} \mathrm{Ni}$ yield.

We thank J. Craig Wheeler for helpful comments on a draft of this manuscript, and Rodolfo Barniol Duran, Lars Bildsten, Adam Burrows, Emmanouil Chatzopoulos, and Sean Couch for valuable conversations. The software used in this work was in part developed by the DOE-supported ASC/Alliance Center for Astrophysical Thermonuclear Flashes at the University of Chicago. The authors acknowledge the Texas Advanced Computing Center (TACC) at the University of Texas at Austin for providing high-performance computing resources that have contributed to this research. C.C.L. acknowledges support from a National Science Foundation Graduate Research Fellowship. M.M. acknowledges support from NSF grants AST-0708795 and AST-1009928. P.K. acknowledges support from NSF grant AST-0909110.

\section{REFERENCES}

Arnett, W. D. 1987, ApJ, 319, 136

Arnett, W. D., \& Meakin, C. 2011, ApJ, 733, 78

Artemova, I. V., Bjoernsson, G., \& Novikov, I. D. 1996, ApJ, 461, 565

Balbus, S. A., \& Hawley, J. F. 1998, Rev. Mod. Phys., 70, 1

Balbus, S. A., \& Hawley, J. F. 2002, ApJ, 573, 749

Beloborodov, A. M. 1999, in ASP Conf. Ser. 161, High Energy Processes in Accreting Black Holes, ed. J. Poutanen \& R. Svensson (San Francisco, CA: ASP), 295

Beloborodov, A. M. 2003, ApJ, 588, 931

Bethe, H. A., \& Wilson, J. R. 1985, ApJ, 295, 14

Binney, J., \& Tremaine, S. (ed.) 2008, Galactic Dynamics (2nd ed.; Princeton, NJ: Princeton Univ. Press)

Bisnovatyi-Kogan, G. S. 1971, SvA, 14, 652

Bisnovatyi-Kogan, G. S. 2001, in Stellar Physics. Vol. 1: Fundamental Concepts and Stellar Equilibrium, ed. A. Y. Blinov \& M. Romanova (Astronomy and Astrophysics Library, Translated from the Russian; Berlin: Springer), 323 
Blandford, R. D., \& Begelman, M. C. 1999, MNRAS, 303, L1

Blandford, R. D., \& Begelman, M. C. 2004, MNRAS, 349, 68

Bloom, J. S., Kulkarni, S. R., Price, P. A., et al. 2002, ApJ, 572, L45

Bucciantini, N., Quataert, E., Arons, J., Metzger, B. D., \& Thompson, T. A. 2007, MNRAS, 380, 1541

Bucciantini, N., Quataert, E., Arons, J., Metzger, B. D., \& Thompson, T. A. 2008, MNRAS, 383, L25

Bucciantini, N., Quataert, E., Metzger, B. D., et al. 2009, MNRAS, 396, 2038 Buras, R., Janka, H.-T., Rampp, M., \& Kifonidis, K. 2006a, A\&A, 457, 281

Buras, R., Rampp, M., Janka, H.-T., \& Kifonidis, K. 2006b, A\&A, 447, 1049

Burrows, A. 1986, ApJ, 300, 488

Burrows, A., Dessart, L., Livne, E., Ott, C. D., \& Murphy, J. 2007, ApJ, 664, 416

Calder, A. C., Townsley, D. M., Seitenzahl, I. R., et al. 2007, ApJ, 656, 313

Campana, S., Mangano, V., Blustin, A. J., et al. 2006, Nature, 442, 1008

Chen, W.-X., \& Beloborodov, A. M. 2007, ApJ, 657, 383

Chieffi, A., \& Limongi, M. 2004, ApJ, 608, 405

Chornock, R., Berger, E., Levesque, E. M., et al. 2010, arXiv:1004.2262

Christodoulou, D. M., Contopoulos, J., \& Kazanas, D. 2003, ApJ, 586, 372

Cloutman, L. D., \& Eoll, J. G. 1976, ApJ, 206, 548

Cobb, B. E., Bloom, J. S., Perley, D. A., et al. 2010, ApJ, 718, L150

Della Valle, M., Chincarini, G., Panagia, N., et al. 2006, Nature, 444, 1050

Della Valle, M., Malesani, D., Benetti, S., et al. 2003, A\&A, 406, L33

Dessart, L., Burrows, A., Livne, E., \& Ott, C. D. 2008, ApJ, 673, L43

Dessart, L., Hillier, D. J., Livne, E., et al. 2011, MNRAS, 414, 2985

Di Matteo, T., Perna, R., \& Narayan, R. 2002, ApJ, 579, 706

Fryer, C. L., Young, P., Bennett, M., et al. 2008, arXiv:0811.4648

Fryxell, B., Olson, K., Ricker, P., et al. 2000, ApJS, 131, 273

Fujimoto, S.-i., Kotake, K., Yamada, S., Hashimoto, M.-a., \& Sato, K. 2006, ApJ, 644, 1040

Galama, T. J., Tanvir, N., Vreeswijk, P. M., et al. 2000, ApJ, 536, 185

Galama, T. J., Vreeswijk, P. M., van Paradijs, J., et al. 1998, Nature, 395, 670

Garnavich, P. M., Stanek, K. Z., Wyrzykowski, L., et al. 2003, ApJ, 582, 924

Hamuy, M. 2003, ApJ, 582, 905

Heger, A., Fryer, C. L., Woosley, S. E., Langer, N., \& Hartmann, D. H. 2003, ApJ, 591,288

Hjorth, J., \& Bloom, J. S. 2011, in Gamma-Ray Bursts, ed. C. Kouveliotou, R. A. M. J. Wijers, \& S. E. Woosley (Cambridge: Cambridge Univ. Press), chapter 9 , in press

Hjorth, J., Sollerman, J., Mller, P., et al. 2003, Nature, 423, 847

Igumenshchev, I. V. 2002, ApJ, 577, L31

Igumenshchev, I. V., Abramowicz, M. A., \& Narayan, R. 2000, ApJ, 537, L27

Igumenshchev, I. V., Narayan, R., \& Abramowicz, M. A. 2003, ApJ, 592, 1042

Iwamoto, K., Mazzali, P. A., Nomoto, K., et al. 1998, Nature, 395, 672

Joggerst, C. C., Almgren, A., Bell, J., et al. 2010, ApJ, 709, 11

Jones, E. M., Smith, B. W., \& Straka, W. C. 1981, ApJ, 249, 185

Kasen, D., \& Woosley, S. E. 2009, ApJ, 703, 2205

Kawabata, K. S., Deng, J., Wang, L., et al. 2003, ApJ, 593, L19

Khokhlov, A. M. 1991, A\&A, 245, 114

Kohri, K., Narayan, R., \& Piran, T. 2005, ApJ, 629, 341

Kuhfuß, R. 1986, A\&A, 160, 116

Kumar, P., Narayan, R., \& Johnson, J. L. 2008a, Science, 321, 376

Kumar, P., Narayan, R., \& Johnson, J. L. 2008b, MNRAS, 388, 1729

Landau, L. D., \& Lifshitz, E. M. 1959, Fluid Mechanics (Oxford: Pergamon)

Larsson, J., Levan, A. J., Davies, M. B., \& Fruchter, A. S. 2007, MNRAS, 376, 1285

Lazzati, D., Blackwell, C. H., Morsony, B. J., \& Begelman, M. C. 2011a, MNRAS, 411, L16

Lazzati, D., Morsony, B. J., \& Begelman, M. C. 2010, ApJ, 717, 239

Lazzati, D., Morsony, B. J., \& Begelman, M. C. 2011b, ApJ, 732, 34

Lee, W. H., \& Ramirez-Ruiz, E. 2006, ApJ, 641, 961

Limongi, M., \& Chieffi, A. 2003, ApJ, 592, 404

Lindner, C. C., Milosavljević, M., Couch, S. M., \& Kumar, P. 2010, ApJ, 713, 800

López-Cámara, D., Lee, W. H., \& Ramirez-Ruiz, E. 2009, ApJ, 692, 804

López-Cámara, D., Lee, W. H., \& Ramirez-Ruiz, E. 2010, ApJ, 716, 1308

MacFadyen, A. I., \& Woosley, S. E. 1999, ApJ, 524, 262

MacFadyen, A. I., Woosley, S. E., \& Heger, A. 2001, ApJ, 550, 410

Maeda, K., \& Tominaga, N. 2009, MNRAS, 394, 1317
Malesani, D., Tagliaferri, G., Chincarini, G., et al. 2004, ApJ, 609, L5

Marek, A., \& Janka, H.-T. 2009, ApJ, 694, 664

Matheson, T., Garnavich, P. M., Stanek, K. Z., et al. 2003, ApJ, 599, 394

Mazzali, P. A., Deng, J., Pian, E., et al. 2006, ApJ, 645, 1323

Mazzali, P. A., Deng, J., Tominaga, N., et al. 2003, ApJ, 599, L95

McKinney, J. C., \& Gammie, C. F. 2002, ApJ, 573, 728

Metzger, B. D. 2012, MNRAS, 419, 827

Mezzacappa, A., Bruenn, S. W., Blondin, J. M., Hix, W. R., \& Bronson Messer, O. E. 2007, in AIP Conf. Ser. 924, The Multicolored Landscape of Compact Objects and Their Explosive Origins (Melville, NY: AIP), 234

Milosavljević, M., Lindner, C. C., Shen, R., \& Kumar, P. 2012, ApJ, 744, 103

Mirabal, N., Halpern, J. P., An, D., Thorstensen, J. R., \& Terndrup, D. M. 2006, ApJ, 643, L99

Modjaz, M., Stanek, K. Z., Garnavich, P. M., et al. 2006, ApJ, 645, L21

Morsony, B. J., Lazzati, D., \& Begelman, M. C. 2007, ApJ, 665, 569

Morsony, B. J., Lazzati, D., \& Begelman, M. C. 2010, ApJ, 723, 267

Murphy, J. W., \& Burrows, A. 2008, ApJ, 688, 1159

Murphy, J. W., \& Meakin, C. 2011, ApJ, 742, 74

Nagakura, H., Ito, H., Kiuchi, K., \& Yamada, S. 2011a, ApJ, 731, 80

Nagakura, H., Suwa, Y., \& Ioka, K. 2011b, arXiv:1104.5691

Nagataki, S., Mizuta, A., \& Sato, K. 2006, ApJ, 647, 1255

Narayan, R., \& Yi, I. 1994, ApJ, 428, L13

Narayan, R., \& Yi, I. 1995, ApJ, 444, 231

Nordhaus, J., Burrows, A., Almgren, A., \& Bell, J. 2010, ApJ, 720, 694

Nousek, J. A., Kouveliotou, C., Grupe, D., et al. 2006, ApJ, 642, 389

O'Brien, P. T., Willingale, R., Osborne, J., et al. 2006, ApJ, 647, 1213

O’Connor, E., \& Ott, C. D. 2010, Class. Quantum Grav., 27, 114103

Paczyński, B., \& Wiita, P. J. 1980, A\&A, 88, 23

Perna, R., \& MacFadyen, A. 2010, ApJ, 710, L103

Pian, E., Mazzali, P. A., Masetti, N., et al. 2006, Nature, 442, 1011

Podsiadlowski, P., Mazzali, P. A., Nomoto, K., Lazzati, D., \& Cappellaro, E. 2004, ApJ, 607, L17

Popham, R., Woosley, S. E., \& Fryer, C. 1999, ApJ, 518, 356

Pruet, J., Thompson, T. A., \& Hoffman, R. D. 2004, ApJ, 606, 1006

Pruet, J., Woosley, S. E., \& Hoffman, R. D. 2003, ApJ, 586, 1254

Raskin, C., Scannapieco, E., Rhoads, J., \& Della Valle, M. 2008, ApJ, 689, 358

Reichart, D. E. 1999, ApJ, 521, L111

Ryu, D., \& Goodman, J. 1992, ApJ, 388, 438

Scheck, L., Kifonidis, K., Janka, H.-T., \& Müller, E. 2006, A\&A, 457, 963

Seitenzahl, I. R., Timmes, F. X., Marin-Laflèche, A., et al. 2008, ApJ, 685, L129

Sekiguchi, Y., \& Shibata, M. 2011, ApJ, 737, 6

Shakura, N. I., \& Sunyaev, R. A. 1973, A\&A, 24, 337

Shigeyama, T., Nomoto, K., Hashimoto, M., \& Sugimoto, D. 1987, Nature, 328, 320

Smartt, S. J. 2009, ARA\&A, 47, 63

Soderberg, A. M., Nakar, E., Berger, E., \& Kulkarni, S. R. 2006, ApJ, 638, 930

Stanek, K. Z., Matheson, T., Garnavich, P. M., et al. 2003, ApJ, 591, L17

Starling, R. L. C., Wiersema, K., Levan, A. J., et al. 2011, MNRAS, 411, 2792

Stone, J. M., \& Balbus, S. A. 1996, ApJ, 464, 364

Stone, J. M., Pringle, J. E., \& Begelman, M. C. 1999, MNRAS, 310, 1002

Surman, R., McLaughlin, G. C., \& Hix, W. R. 2006, ApJ, 643, 1057

Tagliaferri, G., Goad, M., Chincarini, G., et al. 2005, Nature, 436, 985

Taylor, P. A., Miller, J. C., \& Podsiadlowski, P. 2011, MNRAS, 410, 2385

Thielemann, F.-K., Hashimoto, M.-A., \& Nomoto, K. 1990, ApJ, 349, 222

Thompson, T. A., Chang, P., \& Quataert, E. 2004, ApJ, 611, 380

Thompson, T. A., Quataert, E., \& Burrows, A. 2005, ApJ, 620, 861

Timmes, F. X., \& Swesty, F. D. 2000, ApJS, 126, 501

Wheeler, J. C., Yi, I., Höflich, P., \& Wang, L. 2000, ApJ, 537, 810

Woosley, S. E. 1993, ApJ, 405, 273

Woosley, S. E., \& Bloom, J. S. 2006, ARA\&A, 44, 507

Woosley, S. E., \& Heger, A. 2006, ApJ, 637, 914

Woosley, S. E., \& MacFadyen, A. I. 1999, A\&AS, 138, 499

Woosley, S. E., Pinto, P. A., \& Ensman, L. 1988, ApJ, 324, 466

Woosley, S. E., \& Weaver, T. A. 1995, ApJS, 101, 181

Woosley, S. E., \& Weaver, T. A. 1986, ARA\&A, 24, 205

Young, P. A., \& Fryer, C. L. 2007, ApJ, 664, 1033

Zhang, W., Woosley, S. E., \& Heger, A. 2008, ApJ, 679, 639

Zimmerman, E. R., Narayan, R., McClintock, J. E., \& Miller, J. M. 2005, ApJ, 618,832 